\title{
Hybrid Dynamic Route Planning Model for Pedestrian Microscopic Simulation at Subway Station
}

\author{
Yongxin Gao $\mathbb{D}^{1},{ }^{1}$ Feng Chen $\mathbb{D},{ }^{1,2}$ and Zijia Wang $\mathbb{D}^{1}$ \\ ${ }^{1}$ School of Civil and Architectural Engineering, Beijing Jiaotong University, Beijing, China \\ ${ }^{2}$ School of Highway, Chang'an University, Xian, China \\ Correspondence should be addressed to Yongxin Gao; 13115312@bjtu.edu.cn and Zijia Wang; zjwang@bjtu.edu.cn
}

Received 26 December 2018; Revised 19 March 2019; Accepted 26 March 2019; Published 17 April 2019

Guest Editor: Emiliano Cristiani

Copyright (c) 2019 Yongxin Gao et al. This is an open access article distributed under the Creative Commons Attribution License, which permits unrestricted use, distribution, and reproduction in any medium, provided the original work is properly cited.

\begin{abstract}
To make agents' route decision-making behaviours as real as possible, this paper proposes a layered navigation algorithm, emphasizing the coordinating of the global route planning at strategic level and the local route planning at tactical level. Specifically, by an improved visibility graph method, the global route is firstly generated based on static environment map. Then, a new local route planning (LRP) based on dynamic local environment is activated for multipath selection to allow pedestrian to respond changes at a real-time sense. In particular, the LRP model is developed on the basis of a passenger's psychological motivation. The pedestrians' individual preferences and the uncertainties existing in the process of evaluation and choice are fully considered. The suitable local path can be generated according to an estimated passing time. The LRP model is applied to the choice of ticket gates at a subway station, and the behaviours of gate choosing and rechoosing are investigated. By utilizing $\mathrm{C}++$, the layered navigation algorithm is implemented. The simulation results exhibit agents' tendency to avoid congestion, which is often observed in real crowds.
\end{abstract}

\section{Introduction}

Pedestrian simulation has engaged the attention of researchers over the past few decades. It is not only a simulation technology to reproduce the temporal and spatial changes of pedestrian flow in real scenes, but also an important application of computer technology in traffic studies. Different technical areas, such as architecture, urban planning, and transportation, can benefit from the simulation of groups of pedestrians. The simulation can help to improve the efficiency, comfort, and safety of pedestrian transport, to provide references for planning, design, and facility layout, or to optimize passenger flow organization and emergency evacuation plan.

The framework used for describing pedestrian traffic can be specified in a three-tier structure [1]. At the highest strategic level, the pedestrian agents generate the global route according to the activity planning and the topological network of building environment. The tactical level is performed in case of multipath choice. At the operational level, microscopic motions (e.g., walking in an area and moving in a queue) can be observed. To our knowledge, the development of behaviour models at the strategic level in the existing literature is fairly robust $[2,3]$ and a great deal of research has been dedicated towards modeling the operational-level movement of pedestrians $[4,5]$. However, the intermediate tactical level is usually neglected. Generally, most global path planning (GRP) is static and cannot adjust to the changes of environment. Although some studies adopted a real-time global path planning [6], it always means a large amount of computing resources consumption. Though the operational-level navigation can take full account of the dynamic environment, but it is difficult to ensure an optimal route from an overall perspective due to the limited local information obtained by the agents. Therefore, to make a balance of efficiency and flexibility, this paper proposes a hybrid path planning model, which combines GRP at strategic level with local route planning (LRP) at tactical level. As shown in Figure 1, during the journey from starting point to destination, a pedestrian is guided by a series of target 


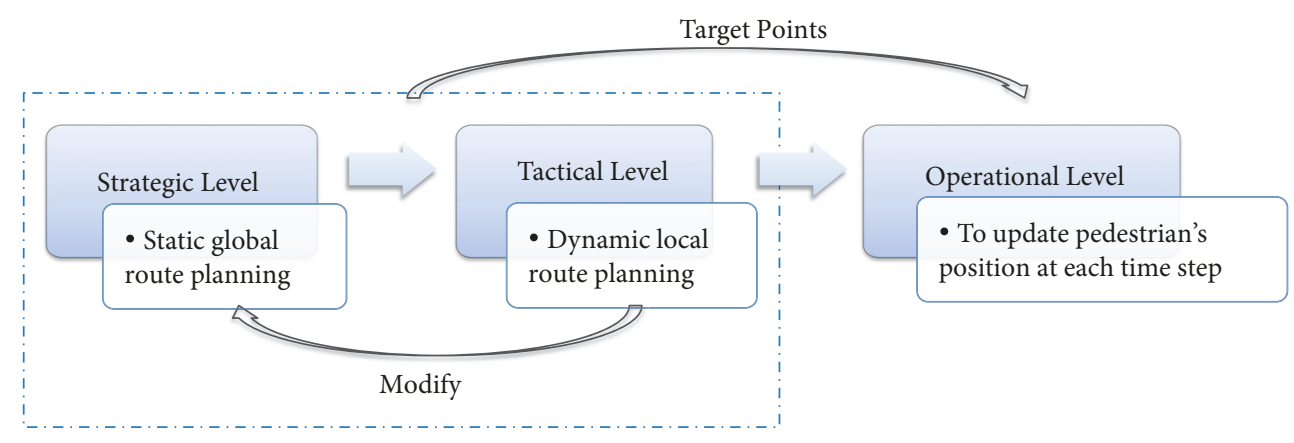

FIgURE 1: The three levels of navigation algorithm.

points. These target points are generated by GRP based on the static building environment and dynamically modified by the LRP with the changing surroundings.

For the behaviour of multipath selection at tactical level, utility and probability are often used to solve this problem. Some researches defined the utility as time [7] or a function of some factors of the path such as distance, excitement, and density [8]. Reference [9] defined the utility as a sum of the relative values of distance and density. Paper [7] evaluated the exit choice by the maximum of the distance-based time and density-based time. In these studies, the route with most utility or least disutility will be definitely chosen. However, the choice of a pedestrian is not always sufficiently sensible. They cannot always make accurate estimations and optimal choices. To reflect the randomness of path choice, some researches introduced probability into this field. Reference [10] expressed the probability of selecting a certain path as a combination of the reliability probability and the cognitive probability. Paper [11] evaluated the probability of exit choice by a weighted sum of the distance-based probability and the density-based probability. The weights depend on the degree of deviation of the alternative paths. This model was also applied to the selection of ticket gates in subway stations. Reference [12] calculated the probability by an exponential function of distance and queue number. Among the studies attempting to model route choice behaviour, the Logit model based on the utility of each route [13] has been proved as a popular and practical approach. The mixed nested Logit model [14], multinomial Logit model [15], and other Logit models with complex forms have been proposed to estimate the behaviour of multipath choice. References $[16,17]$ constructed the utility of a path by a linear weighted sum of distance and time. In some studies, the time factor was replaced by density [18]. Paper [16] suggested that pedestrians' preferences for the factors are related to their speed. Reference [19] proposed a node selection model of ticket gates at metro stations and the linear weighting utility consists of multiple factors. Paper [14] evaluated the cost by a sum of distance-based time and density-based time. Unfortunately, the development of probability and generalized utility in these studies are usually the results of mathematical matching with multiple factors and lack of practical physical significance. This is not conducive to explaining the fundamental motivation of pedestrians to make choices and may lead to the deviation between the simulation results and the actual situation. Consequently, this paper aims to model the individual desires and interests of multipath choice to generate realistic community behaviours.

In the literature, the passenger simulation of multipath choice behaviour aims to achieve two purposes. One purpose is to explore the behaviour pattern of pedestrians in reality and then reproduce and estimate their selections. Some experiments have been made to explain the factors that affect pedestrians' choices, such as density, distance, visibility [20], and social influence $[21,22]$. There are also data collected by stated-choice investigation $[23,24]$ and video [17] used to analyze passengers' selection behaviour. Another purpose is to find out the optimal guidance scheme of the pedestrians to each path to get a utility balance or to get the least evacuation time $[25,26]$. This is often used in the layout design and evacuation organization. Paper [27] designed a class of variable guide sign systems based on the dynamic balance of each exit. Obviously, the former purpose is the foundation of the latter. Only by understanding the mechanism of pedestrian selection behaviour, the appropriate guidance management can be made. Therefore, this paper aims to reveal the mechanism of pedestrian choice behaviour in terms of psychology.

In design and operation stages of metro system, pedestrian simulation has become a necessary, low-cost, and effective method to improve facilities layout and passenger organization. It is of great significance to ensure the safety and good performance in operation. In order to achieve a reliable simulation result, the accuracy of simulation models and parameters are particularly important. At present, there are some commercial software aiming to simulate passenger behaviour, such as Pathfinder, Exodus, and PTV Viswalk. These tools have different characteristics and their own specific application range. Although some standardized verification and validation documents are published as a reference, these documents are directed at building environment, which is not necessarily used for subway scenes. This motivated the development of dedicated models and more accurate knowledge of pedestrian movement at different locations and facilities in subway. In particular, the multipath choice behaviour in subway stations at ticket gates [11, 19], stairs/escalator [28], and the waiting position on platform [29] is modeled in some researches. Similarly, the passengers' 
motion during the check-in process in a high speed railway station is also studied in [30]. However, the whole process of ticket gate selection is not completely explored. This paper attempts to make up this gap. The behaviour of ticket gate selection is decomposed into several stages and embedded in the navigation model.

The multipath selection occurs when a pedestrian faces multiple paths [26], exits, and intermediate nodes. Nevertheless, the selection of multiple intermediate nodes, such as the ticket gates in a subway station, is different in some details from the other two kinds of multipath choices. Specifically, in order to avoid interference, the exits are usually dispersed in space. Differently, intermediate nodes such as service windows and ticket gates are usually centralized. As a result, all the nodes are visible to pedestrians in geometric space. Different from the exits, the intermediate nodes are not the end of trip. So, rather than just focusing on the condition before passing the nodes, the condition after passing, such as the distance between the nodes to the next target point, should also be taken into account. Furthermore, the node selection may involve some activities like queuing and ticket checking. Therefore, as pedestrians would adopt different behaviours to deal with these three kinds of multipath situations, it is inappropriate to model them by the same model. For example, there are some unreasonable assumptions when applied the exit-choice model to the selection of ticket gates [11]. For this reason, some necessary adjustments are made to the scalable LRP method proposed in this paper so as to apply it to the selection behaviour of ticket gates.

Uncertainty always exists in system components and processes, which are normally resulting from uncertain cost in nature and deviations in subjective judgments. Such a variety of uncertainties may become more compounded by combinations of uncertain information presented in multiple forms. Previously, a number of research works for uncertain methods in traffic simulation were undertaken. Reference [31] pointed out the effect of environmental uncertainty on cooperation of evacuation crowd and evacuation efficiency. Papers [32,33] optimized the evacuation organization when the demand uncertainty and capacity uncertainty were taken into account. Actually, uncertainty also exists in many aspects of pedestrian path selection. For instance, since pedestrians have the characteristics of rational irrationality and randomness, they do not always make the best choice [34] and make the same decisions under the same circumstances. In addition, pedestrians have different preferences of choosing path. Their perceptions of the distance and the social influence factors are different [21]. Reference [35] pointed out that the evacuees could be divided into the patient and the impatient. The patient evacuees try to avoid physical contacts, but the impatient evacuees vie for the empty target cell by paying the effort. Similarly, [36] divided them into the active and the conservative. Reference [12] even considered four types of window choice strategies. It has been proved that the uncertainties will affect the results of path selection, but they have not been explicitly addressed in the existing literature. In this paper, the uncertainty underlying in route planning will be taken into account in three ways: the estimation of factors, the randomness of decision-making, and the subjective individual preference.

In this paper, a three-level navigation algorithm is proposed to capture passenger movement in subway station. Especially, the improved pedestrian route planning model balances the certainty of global route planning at the strategic level and the flexibility of local route planning at the tactical level. The local route planning model is developed based on a passenger's psychological motivation. The uncertainties and preferences within the process of path selection are fully considered. With this navigation algorithm, the most appropriate path will be activated according to real-time environment. The difference between simulation results and real situation can be minimized.

The study begins with the method of abstracting route planning network from realistic environment in Section 2.1 and generating a collision-free global route in Section 2.2. Following, a novel local route algorithm is described in Section 2.3. The field data collected in actual subway stations is given in Section 3.1. Then, an application to the selection of ticket gates is provided with some necessary adjustments in the following parts of Section 3. Furthermore, the advantages of the proposed models are demonstrated with examples in Section 4, and, last, Section 5 concludes with a summary and an outlook for future work.

\section{Model}

2.1. Environment Map Modeling. Before route planning, environment map should be established to mark the location of pedestrian and obstacles. Through expressing the obstacles as abstract geometric figures, the walking area in real world can be translated into perceivable environment information for pedestrians.

The common methods to model environment are obstacles polygon, barrier-free zone, hybrid space, and path diagram $[37,38]$. Since pedestrians in subway station only need to recognize obstacles to avoid collision, this paper adopts obstacles polygon method to divide the station environment into two regions: walking area and obstacle area. Obstacle areas are abstracted as approximate polygons. All the other areas except obstacles are considered as walking areas on the basis of continuum space model.

\subsection{Global Route Planning Model Based on Visibility Graph.} Global route planning is indispensable in a pedestrian simulation. It can optimize the layout of guidance facilities, reduce calculating quantity, and decrease errors caused by manual operation, especially in an environment with lots of obstacles. The main route planning algorithms are visibility graph, free space and grids. The complexity of free space method increases with the amount of obstacles dramatically and this method cannot find the shortest path in any cases. The stored environment information of grids method is huge and the suitable grid size is difficult to determine. If gird size is too small, the calculated amount will be too large while the shortest path may not be got with too big grid. In the literature, most algorithms for global planning represent the 


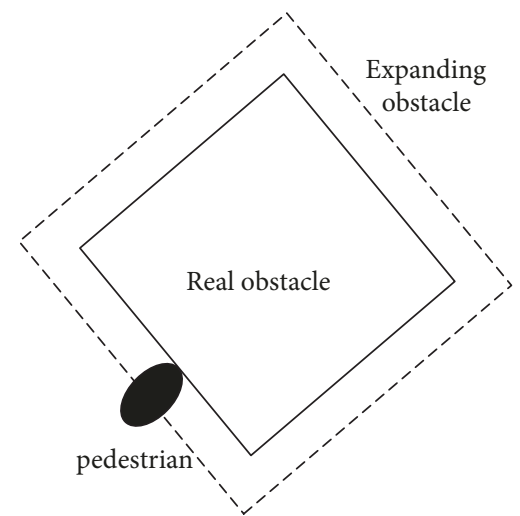

Figure 2: Obstacle expanding illustration.

connectivity of walkable space in the environment as a graph and perform search queries for each agent to determine a collision-free path. We do not diverge from previous work in this aspect and adopt visibility graph method to plan global route.

According to the method of visibility graph, the realistic environment is replaced by approximate polygon in proportion and the visible graph is composed by connecting all nocross and unscreened lines. By using $A *$ algorithm [39], the shortest path between origin and destination can be found on the visible graph, which is the global navigation route.

In global route, all of intermediate points except origin and destination are regarded as convex vertex of obstacles. In traditional visibility graph method, a pedestrian is considered as a point. While in fact, he occupies an area. As a result, a passenger overlaps with margin of obstacles when he walks on the global route [40]. To avoid collision, this paper makes a correction to the intermediate points using half of pedestrian maximum size as shown in Figure 2. The virtual obstacles are used to calculate a global route.

\subsection{Local Route Planning Model Based on Estimated Travel} Time. Global route planning is used to find out the whole route from origin to destination. If there are multiple routes, the shortest path in terms of Euclidean distance is searched. However, when the pedestrian density is large, it will cause unrealistic walking behaviour. For example, during pedestrian passing a ticket gate at a subway station, global route planning strategy will determine a fixed gate; therefore, pedestrians will wait in line at this gate and the other gates will be left unused. However, according to observation and video analysis of pedestrians' route choice behaviour at metro stations, pedestrians will choose a farther unoccupied gate to save travel time in practice. The local route planning model proposed in this paper is made in order to solve this problem.

A pedestrian makes the route choice decision according to what has been seen in the surroundings and what has been formulated in his or her mind. Specifically, environmental factors include path distance or travel time, directness of path, capacity/width of exit, visibility and service level [16, 41]. The individual implicit factors include physical ability (walking speed), subjective preference, familiarity with the environment, and following behaviour [42]. From a review of the previous studies, three factors, namely, the route length, passing time, and density of pedestrian flow, are identified to have significant impacts on a pedestrian's behaviour of route choice [43]. The route length is the primary factor that affects route choice behaviour and the crowdedness is a measure to reflect discomfort [44]. What is more, it is found that a pedestrian tends to base his exit choice on time-dependent information rather than on time-independent information $[9,45]$. Therefore, the local route planning model proposed in this paper allows pedestrians choose a path according to the estimated passing time, which is determined by the length and the crowdedness of each local path, shown as (1) and (2). The local route planning for each pedestrian is independent and dynamic. Namely, which path will be the optimal depends on the individual and the moment.

$$
\begin{aligned}
& U_{i, \mathrm{j}}=V_{i, \mathrm{j}}+\varepsilon_{i, \mathrm{j}} \\
& V_{i, \mathrm{j}}=\omega_{i, \mathrm{j}}^{N} \bullet V_{i, \mathrm{j}}^{N}+\omega_{i, \mathrm{j}}^{L} \bullet V_{i, \mathrm{j}}^{L}
\end{aligned}
$$

where $U_{i, j}$ is the utility for pedestrian $i$ choosing path $j, i=1,2, \ldots, j=1,2, \ldots$. The random error $\varepsilon_{i, j}$ is independent identically distributed and subject to a Gumbel distribution. $V_{i, \mathrm{j}}$ is a certain item, indicating the estimated passing time by pedestrian. It consists of waiting time and walking time. $V_{i, \mathrm{j}}^{N}, V_{i, \mathrm{j}}^{L}$ is the estimated waiting time and the estimated walking time, respectively. $\omega_{i, j}^{T}$ and $\omega_{i, j}^{L}$ are the perceived weights.

In real-world situations, passengers generally give a glance of the possible routes and make a rough estimation by hunch rather than by accurate calculations when making a route choice. Thus, uncertainty of decision variables should be introduced into the route choice model. To an appropriate level, the variability in time cost and uncertainty could help "blur" the agents' route choices. In this way, it can achieve simulation results that conform well to real situation.

2.3.1. Passengers' Perception of Time. During the process of a pedestrian's journey, on one hand, he needs to walk for a certain distance. The time spent on walking is called "walking time". On the other hand, if there are congestions on the way and the pedestrian need to queue for service, he would have to spend extra "waiting time". According to temperament and time urgency, pedestrians may have different values of time (VOT) on "walking time" and "waiting time". It is observed that there is a strong negative correlation between utility coefficients of distance and density, meaning that an individual who has a strong tendency to avoid congestion is likely to be more indifferent about walking for long distances to reach a further less-congested path, and vice versa [46].

2.3.2. Walking Time. Pedestrian $i$ estimates the walking time of the $j$-th local path as

$$
V_{i, j}^{L}=\frac{\sum L_{i, j}^{m \prime}}{v_{i}}
$$


$v_{i}$ is the desired walking speed of pedestrian $i . \sum L_{i, j}^{m \prime}$ is the estimated total length of local path $j$. If we divide path $j$ into several segments, the real length of segment $m$ is $L_{i, j}^{m} \cdot L_{i, j}^{m \prime}$ is the estimated length and can be calculate as (4). The estimated error is limited within $10 \%$ and $k$ is subject to a Gaussian distribution. As a result, $L_{i, j}^{m}$ is also in a Gaussian distribution, with the average of $L_{i, j}^{m}$ and within range of $\left[0.9 \cdot L_{i, j}^{m}, 1.1 \cdot L_{i, j}^{m}\right]$.

$$
\begin{aligned}
L_{i, j}^{m \prime} & =L_{i, j}^{m}+\varepsilon\left(L_{i, j}^{m}\right) \\
\varepsilon\left(L_{i, j}^{m}\right) & =k \cdot L_{i, j}^{m} \\
k & \sim N(0,0.001),|k| \leq 10 \%
\end{aligned}
$$

It is worth noting that although the value of the estimated length has an error, the order of local paths in length should not be changed. To ensure the right order of estimated distances, we should make a limit to the scope of estimated length. Before estimating the length of segment $m$ for each local path, sort the actual distances from short to long. Then, compare every two adjacent distances. For $L_{i, p}^{m}<L_{i, q}^{m}$, if $1.1 \cdot L_{i, p}^{m}>0.9 \cdot L_{i, q}^{m}$, the estimating range should be adjusted to

$$
\begin{aligned}
& L_{i, p}^{m \prime} \in\left[0.9 * L_{i, p}^{m}, \frac{\left(1.1 * L_{i, p}^{m}+0.9 * L_{i, q}^{m}\right)}{2}\right] \\
& L_{i, q}^{m \prime} \in\left[\frac{\left(1.1 * L_{i, p}^{m}+0.9 * L_{i, q}^{m}\right)}{2}, 1.1 * L_{i, q}^{m}\right]
\end{aligned}
$$

2.3.3. Waiting Time. Waiting time is estimated by the product of the estimated number of passengers in queue $\left(N_{i, j}^{\prime}\right)$ and the average service time $\left(t_{0}\right)$, as shown in (6).

$$
V_{i, j}^{N}=N_{i, j}^{\prime} \bullet t_{0}
$$

Pedestrian $i$ would estimate the number of passengers queuing at local path $j$ as

$$
\begin{gathered}
N_{i, j}^{\prime}=N_{i, j}+\varepsilon\left(N_{i, j}\right) \\
\varepsilon\left(N_{i, j}\right)= \begin{cases}0, & N_{i} \leq 3 \\
\{0, \pm 1\}, & N_{i, j} \in[4,5] \\
\{0, \pm 1, \pm 2\}, & N_{i, j} \geq 6\end{cases} \\
P\left\{\varepsilon\left(N_{i, j}\right)=X_{p}\right\}=\frac{1}{3}, \quad\left(X_{p}=-1,0,1\right) \\
P\left\{\varepsilon\left(N_{i, j}\right)=X_{q}\right\}=\frac{1}{5}, \quad\left(X_{q}=-2,-1,0,1,2\right)
\end{gathered}
$$

$N_{i, j}$ is the actual number of queuing at local path $j$ when pedestrian $i$ makes decision. $\varepsilon\left(N_{i, j}\right)$ is estimation error and it would enlarge as the queuing passenger population increases.
Specifically, in case of no more than three pedestrians queuing, pedestrian $i$ can identify the number accurately. If the number is greater than 3, the estimated error would obey a Discrete Uniform Distribution.

2.3.4. Discrete Choice Model. After the walking time and the waiting time having been estimated, the final piece of the puzzle is how to decide which local path a passenger will pass through. As a problem of discrete choice, we adopt the Logit model. The probability of pedestrian $i$ choosing local path $j$ is

$$
\begin{aligned}
P_{i, j} & =\frac{\exp \left(-V_{i, j}\right)}{\sum_{j=1}^{M} \exp \left(-V_{i, j}\right)}, \\
\sum_{j=1}^{M} P_{i, j} & =1
\end{aligned}
$$

For a completely rational pedestrian, he would choose the most effective path to go through. While in practice, a pedestrian's decision is effected by some random factors, such as emotion and the pedestrians around him. For this reason, a pedestrian will not accurately evaluate the route options, and, as a result, the path with the smallest cost is not always chosen [47]. Therefore, the final decision is determined by a random number according to the probabilities $P_{i, j}$.

\section{Model Application and Discussion}

Utilizing MFC provided by Microsoft Visual Studio, we developed a pedestrian simulation tool to realize our navigation algorithm. Specifically, to enable agents to autonomously navigate in complex spaces and crowded environment, the tool combines the visibility graph at strategic level, multistage route choice scheme at tactical level, and a variant of Social Force Model at operational level. The local route planning is realized by a target converter and local route settings. A pedestrian can temporarily deviate from the global route, take reasonable detours to optimize his local route, and return to global route after passing the local route region. At the operational level, a pedestrian's movement towards the target point is driven by a resultant force, which is affected by the goal's attraction, barriers, and other agents. The framework of the layered navigation algorithm is shown in Figure 3.

Thanks to the developed simulation tool, the route choices and crowd characteristics estimated by the navigation algorithm can be favorably compared to the actual situations in practice. During simulation, all the agent movements are recorded to a database. Then, it can be analyzed to determine some key performance metrics such as crowd density, journey time, and flow rates. In addition, the simulation can be played back in real time (or faster) to provide a rich visual means for verifying and communicating. Though the proposed navigation algorithm and simulation tool are applicable in many scenarios, the automatic fare gate in subway stations is taken as an example in this work. 


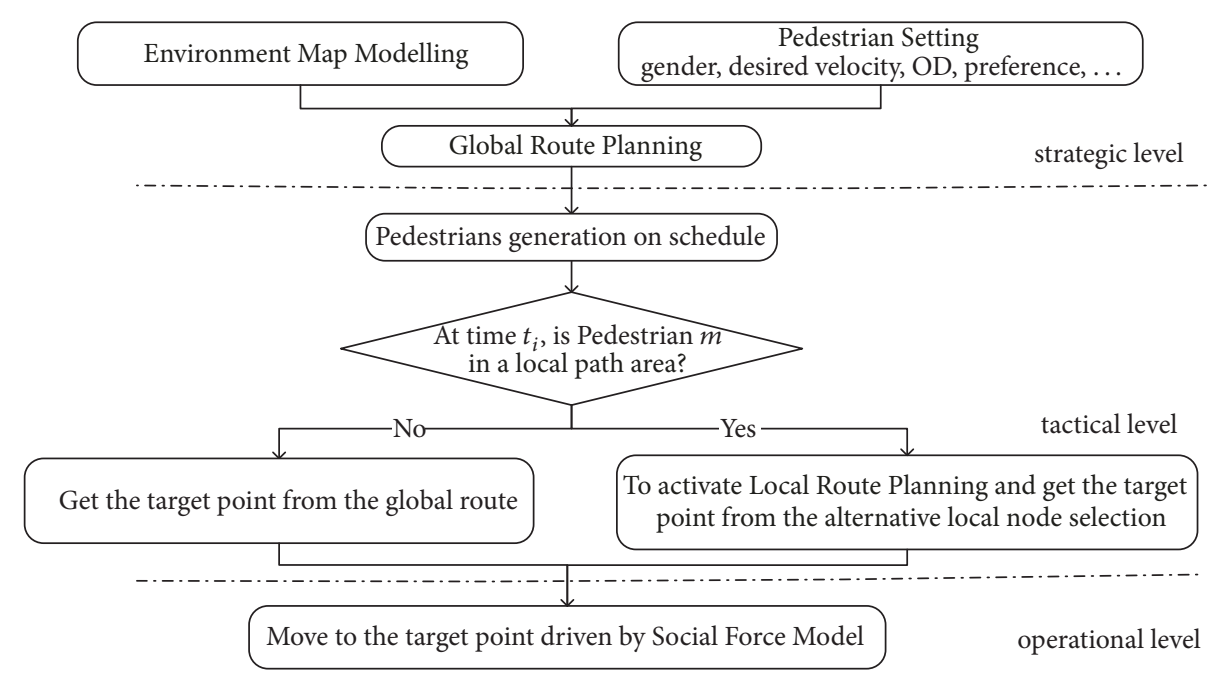

FIGURE 3: The framework of the layered navigation algorithm.

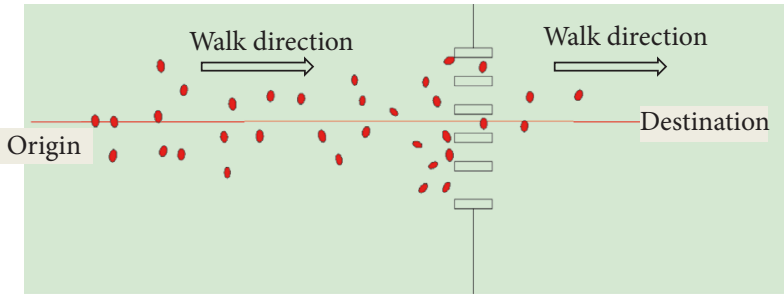

(a)

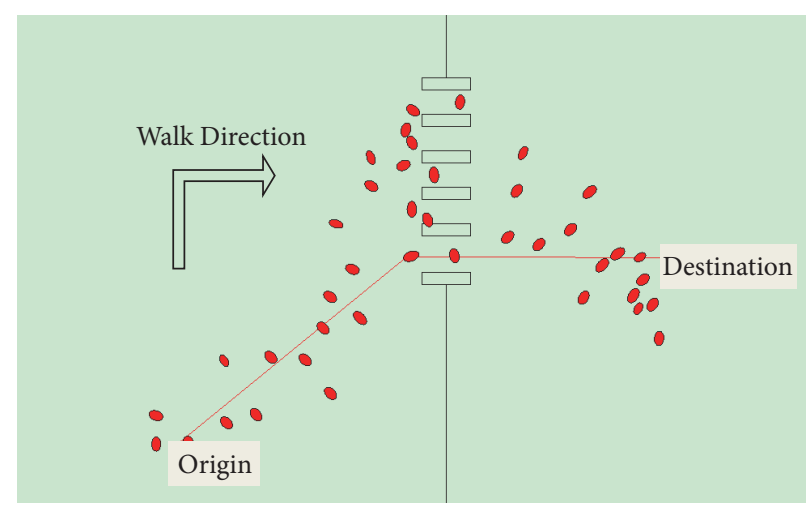

(b)

FIgURE 4: The two simulation scenarios: (a) symmetric layout and (b) asymmetric layout.

3.1. Empirical Data and Simulation Settings. According to field survey in subway stations, it is found that there are different traffic lines because of the spatial layout of ticket gates. Roughly, the situation can be divided into two typical scenarios, i.e., the symmetry and the asymmetry scenario. As shown in Figure 4, in the symmetry scenario, passengers just move forward and pass through the gate directly. In the asymmetry scenario, passengers need to move towards the ticket gates firstly and then make a turn to pass through the gate. In the following sections, the gates are numbered as 1, 2, ..., 5 in turn from top to bottom. At the strategic level, only a fixed one of these gates could be counted as a part of global route. But in practice, a pedestrian would choose the optimal path to replace the default one.

To trigger the local route plan, a virtual influence area is set in front of the ticket gate area where local route planning is needed, as shown in Figure 5. When a pedestrian walks into an influence zone, the local route plan will be activated to substitute global route. The pedestrian's destination will be temporarily reset to the local path's target point marked as $\mathrm{D}$ in Figure 5, which is normally the entrance of a stairway to platform or a turning point of path. Point $\mathrm{O}$ is the realtime location when pedestrians enter into the influence zone. It represents the origin of local path. Points $A_{j}, B_{j}$ are the entrance and the exit of gate $j$. The concentration zones are set to count how many pedestrians are queuing at each gate. The polygonal line $\mathrm{OA}_{\mathrm{j}} \mathrm{B}_{\mathrm{j}} \mathrm{D}$ describes local path $j$. It can be divided into three segments in this scenario: $\mathrm{OA}_{j}, \mathrm{~A}_{\mathrm{j}} \mathrm{B}_{\mathrm{j}}$, and $\mathrm{B}_{j} \mathrm{D}$. Their lengths are $L_{i, j}^{1}, L_{i, j}^{2}$ and $L_{i, j}^{3}$, respectively. Here, $L_{i, j}^{2}$ has the same length for each local path, it can be ignored in calculation. Then, the estimated walking time can be simplified as

$$
V_{i, j}^{L}=\frac{\left(L_{i, j}^{1}{ }^{\prime}+L_{i, j}^{3}{ }^{\prime}\right)}{v_{i}}
$$

In order to get reliable simulation results, it is of great importance to assign the parameters of model with practical values. Generally, in the design of critical facilities, the performance under peak passenger flow is a vital indicator. Based on field data obtained in peak-15-minute of morning 


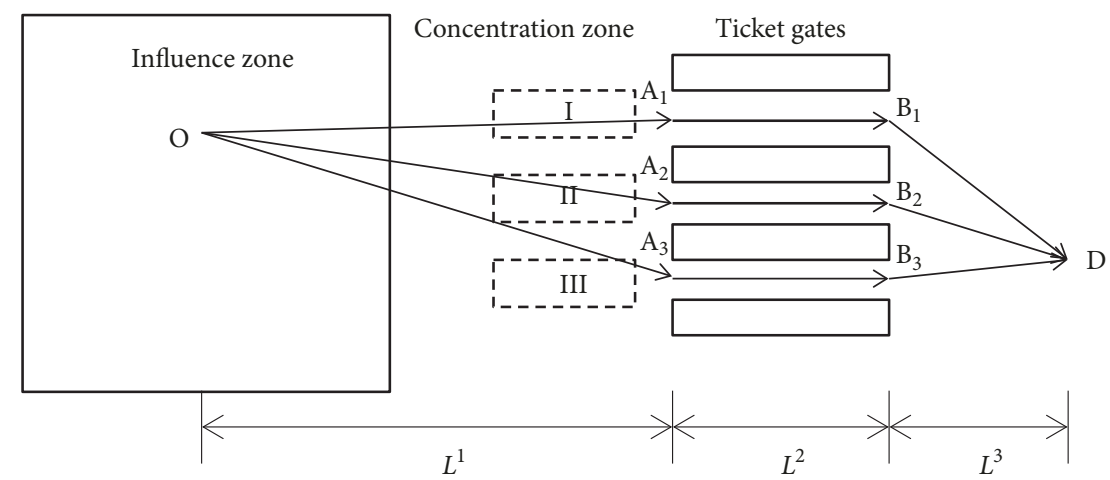

Figure 5: Illustration of local route algorithm.

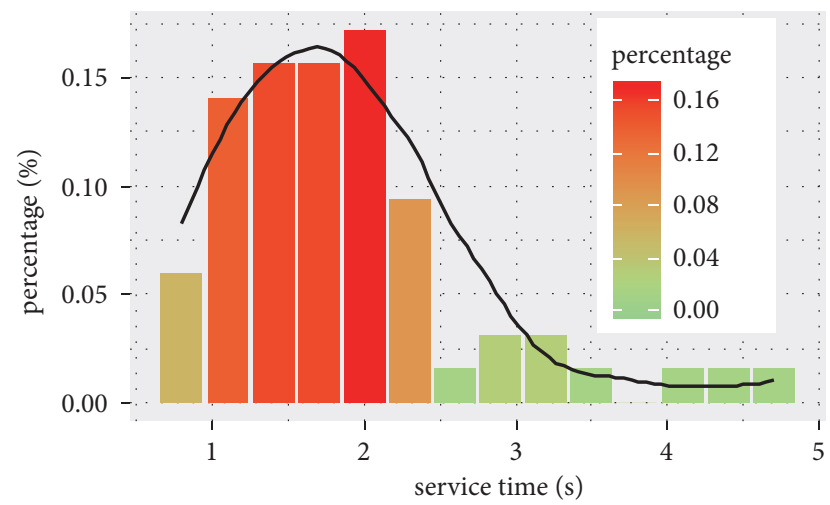

FIgURE 6: The service time of ticket gate based on field survey.

and evening rush hour, passengers' arriving rate at the gate area is subject to Poisson distribution (Equation (10)). The average number of passengers is 40 per minute. The desired speed ranges from $0.8 \mathrm{~m} / \mathrm{s}$ to $1.5 \mathrm{~m} / \mathrm{s}$.

$$
P(X=k)=\frac{\lambda^{k}}{k !} \cdot \exp (-\lambda), \quad k=0,1, \cdots
$$

where $X$ is the arrival interval, $P$ is the probability, and $\lambda$ is a parameter.

The value of service time $t_{0}$ in (6) can be measured by the interval between two passengers entering or leaving a certain ticket gate. Some investigations are carried out in subway stations at morning peak on weekdays. There is heavy passenger traffic during these periods and the passengers are always in a rush to go to work. In the investigation, we recorded the time interval between passengers leaving the end of the gate (point $B_{i}$ in Figure 5) and the results are shown in Figure 6. It is observed that the service time for a pedestrian obeys a positive skewness distribution. It ranges from $0.8 \mathrm{~s}$ to $3.7 \mathrm{~s}$ and the mean value is $2.0 \mathrm{~s}$. So the value of $t_{0}$ in (6) can be regard as 2 seconds.

The time for a passenger passing through the gate (from point $A_{i}$ to $B_{i}$ in Figure 5) is also observed in survey. The results are shown in Figure 7 . More than $80 \%$ passengers can go through $\mathrm{A}_{i} \mathrm{~B}_{i}$ within $1.5 \mathrm{~s} \sim 2.9 \mathrm{~s}$ and the average is $2.4 \mathrm{~s}$. The length of a gate is $1.4 \mathrm{~m}$. According to this, the average speed of a passenger passing through the gate can be obtained. As shown in Figure 7(a), the distribution of passing speed presents a normal distribution with an average of $0.65 \mathrm{~m} / \mathrm{s}$. Interestingly, there is a long tail of the distribution of the passing time (Figure $7(\mathrm{a})$ ), and it disappears by transforming to speed (Figure $7(\mathrm{~b})$ ).

In terms of time perception, the passengers in a subway station can be summarized into three types. The first one is adventurous. They are active and more annoyed with timewaste of standing in a long queue than taking detour for a faster pass. So, their VOT of waiting time is greater. Indeed, commuters in morning peak and passengers who are pressed for time to catch a train or a flight are usually adventurous. In addition, passengers who have higher requirements for a comfortable walking environment are more willing to choose a far path to avoid crowd; they are also adventurous. The second type is the conservative, such as the elderly. Because of their difficulty in moving and other reasons, they prefer a path with shorter walking distance and without bypass. In their views, waiting is much more convenient than walking around. In this case, the VOT of walking time is 1.2 and of waiting time is 0.8 [48]. For some mild passengers, there is no preference of waiting and walking and both could be interpreted as "normal" time. Their perceived time is equivalent to the real time and the VOT is 1 . The perceived weights in (2) can be valued as (11). These values are also referred to simulation experiments. It is the best scheme to keep the simulation results consistent with actual situation.

$$
\begin{gathered}
\omega_{i}^{N}=1.2, \\
\omega_{i}^{L}=0.8,
\end{gathered}
$$

if temperament is adventurous

$$
\begin{gathered}
\omega_{i}^{N}=0.8, \\
\omega_{i}^{L}=1.2,
\end{gathered}
$$

if temperament is conserved

$$
\begin{gathered}
\omega_{i}^{N}=1.0, \\
\omega_{i}^{L}=1.0,
\end{gathered}
$$

if temperament is mild 


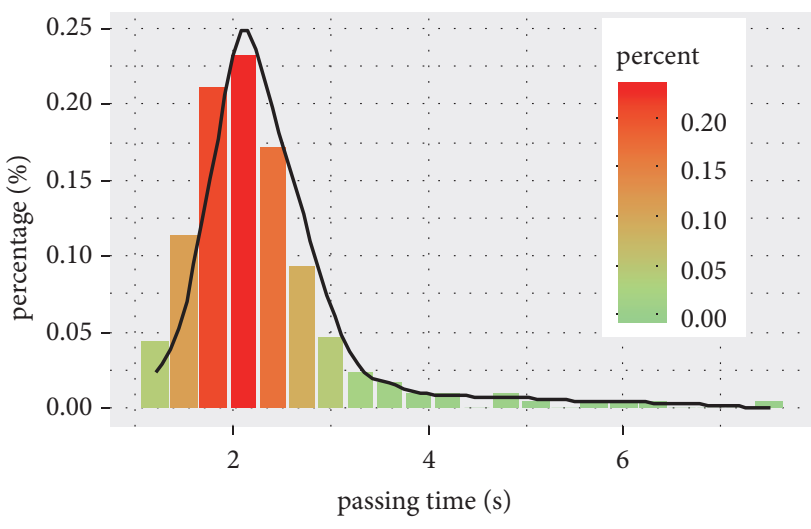

(a)

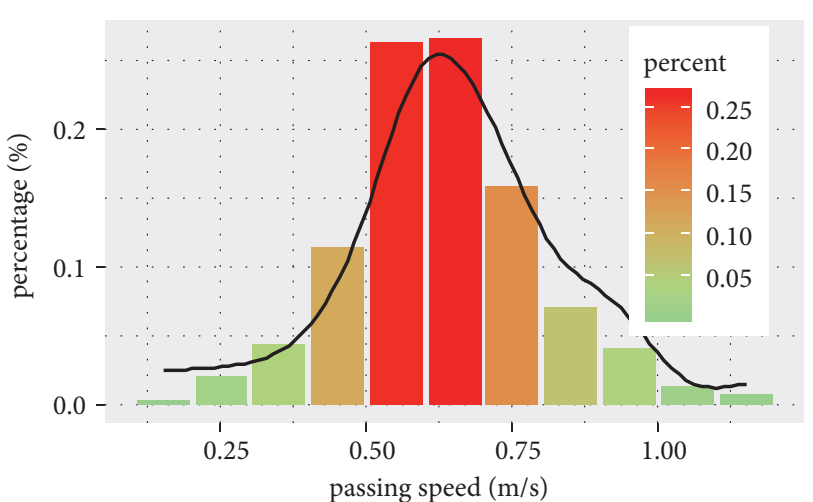

(b)

Figure 7: (a) The time of passing through a gate based on field survey and (b) the calculated passing speed.

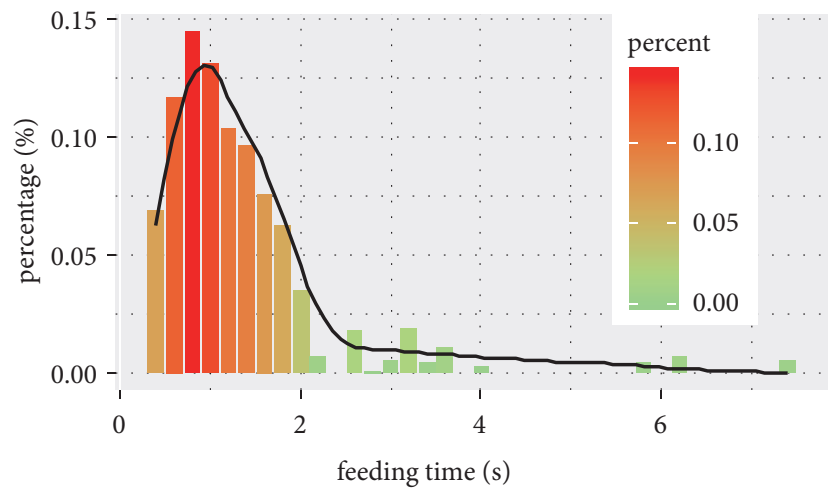

FIGURE 8: The feeding time based on field survey.

To pass through the ticket gate, a special activity must be performed-to check the card. Due to individual habit and the familiarity with subway, the card feeding time of each passenger would be different. In most cases, scanning time ranges from $0.5 \mathrm{~s}$ to $2.0 \mathrm{~s}$ and the average value is $1.1 \mathrm{~s}$, as shown in Figure 8. Occasionally, some greater feeding time can be observed because of a failure to prepare the smart card in advance and improper operation. The maximum value we observed during rush hour is $7.38 \mathrm{~s}$.

The movement of passengers in subway stations and the situations of passing through the ticket gates are also recorded in field survey; the details will be mentioned in the following sections.

3.2. Multistage Route Choice Scheme. Local path selection is a dynamic process. The volume of passengers at each gate is time-varying and the utility value of each local path is also changing. When a pedestrian realizes that the utility value of his original choice obviously increased, or that another path is obviously superior to the original one, he will reevaluate the utility of each path and may change his choice. To solve the problem of reselection, some studies updated the selection choice at each time step [8]. However, this leads to a ping-pong effect [9]. This drawback can be corrected by using inertial system and relaxation processes. With the inertial system, a new selection will be adopted only when it gains a certain increment in contrast to the old selection [7]. With relaxation processes, the selection updates in a given time interval such as 10 seconds $[14,16]$. These methods can achieve good results under certain conditions. However, in a subway system, it is difficult to determine an appropriate threshold for the inertial system and a time interval of reselection for the time-based relaxation processes. Fortunately, according to field studies, the selection process of passing through ticket gates can be abstracted into three stages (Figure 9). Firstly, a pedestrian generally begins to consider which gate is suitable and make a decision in front of the gates with a distance of $3.0 \mathrm{~m}$. Then he moves towards the target gate and always pays attention to the surroundings. In most cases, if necessary, a selection change would be made in front of the gates with a distance of $1.7 \mathrm{~m}$. Next, when he is close to the gate and prepares to check the card, in one case, he may change his choice for the third time. The case is that the passenger in front of him occupancies the gate for a long time and the next gate is unoccupied. The location for the third choice is set in front of the gates at a distance of $1.0 \mathrm{~m}$.

Unlike the first choice, as the pedestrian is considerable close to the gates when he makes the second-time and the third-time route choices, he would pay more attention to the distance between his current position and the gate (i.e., $\mathrm{OA}_{\mathrm{j}}$ ). So the estimated walking time can be simplified to (12). What is more, at that time, the estimations on distance and population size are relatively accurate. He would have more confidence in his judgment and will choose the fastest path, as indicated in (13).

$$
V_{i, j}^{L}=\frac{L_{i, j}^{1}{ }^{\prime}}{v_{i}}
$$

Optimal local path for ped $i$

$$
=\left\{\text { Local Path }_{j} \mid V_{i, j}=\min \left(V_{i, 1}, V_{i, 2}, \cdots V_{i, M}\right)\right\}
$$

As a passenger arrives at the gate, he will stop to press card and then pass through. When a passenger is scanning card, 


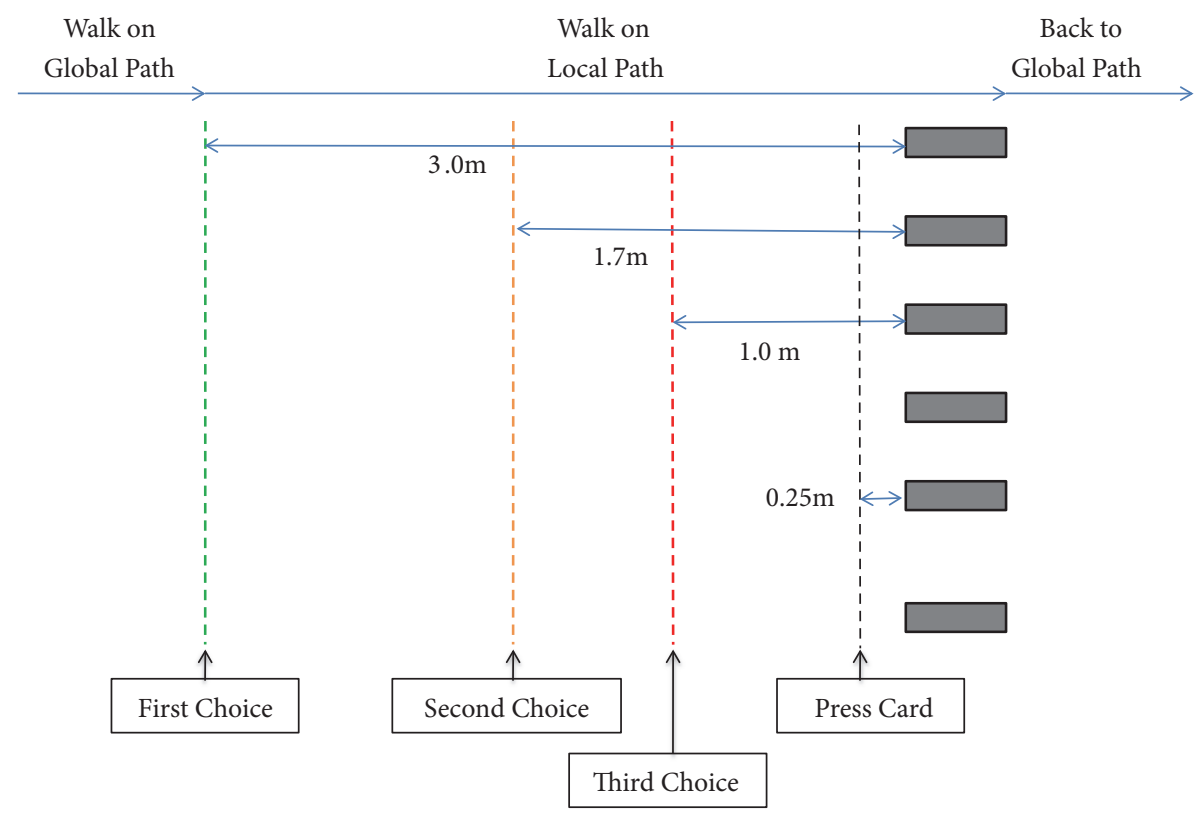

FIgURE 9: The locations to make the multistage route choices.

TABLE 1: Algorithm of local route planning.

\begin{tabular}{|c|c|}
\hline \multirow{3}{*}{ Input } & (a) A set of local path, $J$ \\
\hline & (b) Preference of pedestrian $i, \omega_{i, j}^{N}$ and $\omega_{i, j}^{L}$ \\
\hline & (c) Current speed $v_{i}$ and position $\left(x_{i}, y_{i}\right)$ of pedestrian $i$ at time $t$ \\
\hline Output & A suitable local path for pedestrian $i$ at time $t$ \\
\hline 1 & For each local path $j$ in $J$, do \\
\hline \multirow[b]{2}{*}{2} & ... estimate total walking distance $\sum L_{i, j}^{m \prime}$ by Equations $((4),(5))$. (when pedestrian $i$ \\
\hline & is closed to the gates, $\sum L_{i, j}^{m \prime}$ can be simplified to $L_{i, j}^{1}{ }^{\prime}$ in Equation (12)) \\
\hline 3 & $\ldots \ldots$ calculate walking time $V_{i, j}^{L}$ by Equation $(3)$ \\
\hline 4 & ...estimate the number of passengers queueing $N_{i, j}^{\prime}$ at local path $j$ by Equation (7) \\
\hline 5 & $\ldots \ldots$ calculate waiting time $V_{i, j}^{N}$ by Equation $(6)$ \\
\hline 6 & ...calculate the estimated passing time of path $j$ by Equation (2) \\
\hline 7 & End for \\
\hline 8 & If pedestrian $i$ is closed enough to the ticket gates, then \\
\hline 9 & $\begin{array}{l}\text {..pass through the local path with minimum estimated passing time by Equation } \\
\text { (13) }\end{array}$ \\
\hline 10 & else calculate the probability $P_{i, j}$ of pedestrian $i$ choose local path $j$ by Equation (8) \\
\hline 11 & $\ldots$ choose a suitable local path for pedestrian $i$ based on $P_{i, j}$ and a random number \\
\hline 12 & End if \\
\hline
\end{tabular}

the front edge of his body is almost tangent to the entrance of the gate. So the position of pressing card is set $0.25 \mathrm{~m}$ ahead each gate, as shown in Figure 9.

3.3. Results of Local Route Choice. In order to test the proposed LRP model (the algorithm is summarized in Table 1), two experiments are carried out with the typical scenarios (shown in Figure 4). The ratio of pedestrians with different preferences is assumed as 1:1:1. The simulation lasts for 5 minutes with 200 passengers. In addition, the actual passenger flows of each gate in the two scenarios are also observed in Beijing Subway at peak hours. As shown in Figure 10, it indicates similar passenger distributions at each gate in field investigations and simulation results. Specifically, in the symmetric scenario, most passengers go through the three gates in the middle, which are the shortest paths. While there is less passenger flow of the farthest gates 1 and 5, all the 200 passengers continued to pass through the gates from $12 \mathrm{~s}$ to $342 \mathrm{~s}$ and the average service level of the gates is 36 person/min. Similarly, in the asymmetric scenario, the 


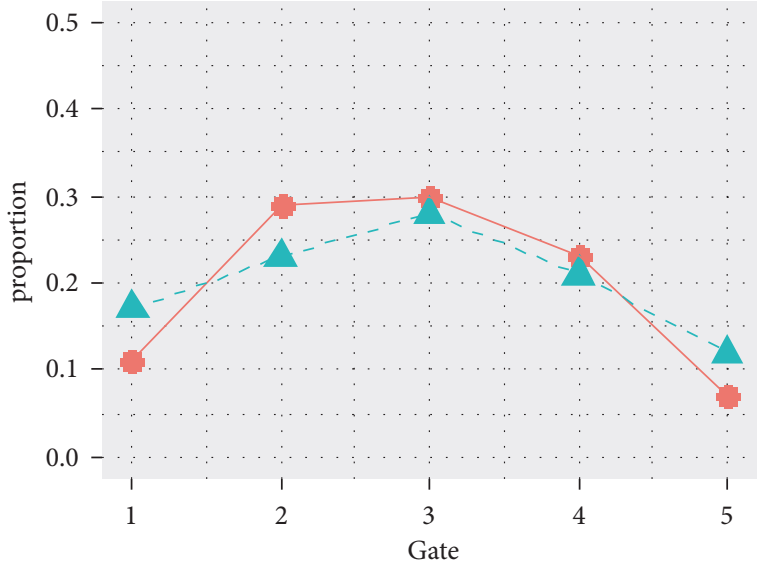

Field Data

Simulation Data

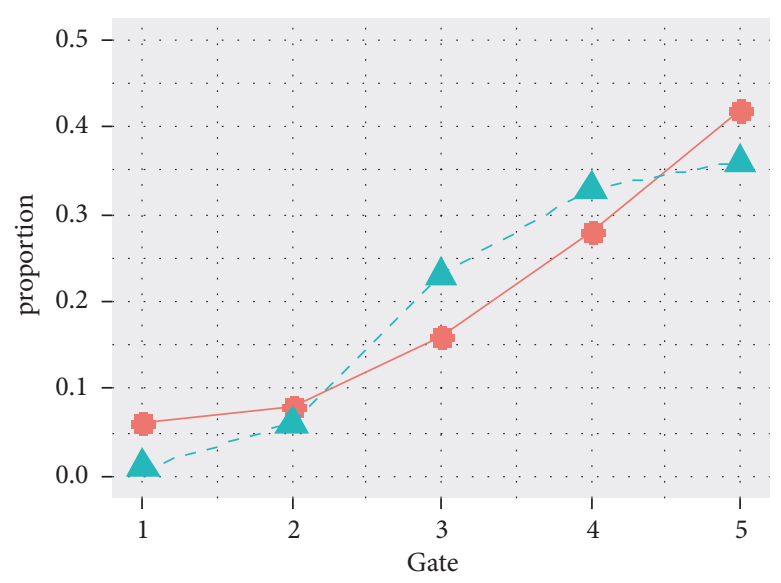

Field Data

Simulation Data

FIGURE 10: Comparison of simulation results and survey data under symmetric layout (left) and asymmetric layout (right).

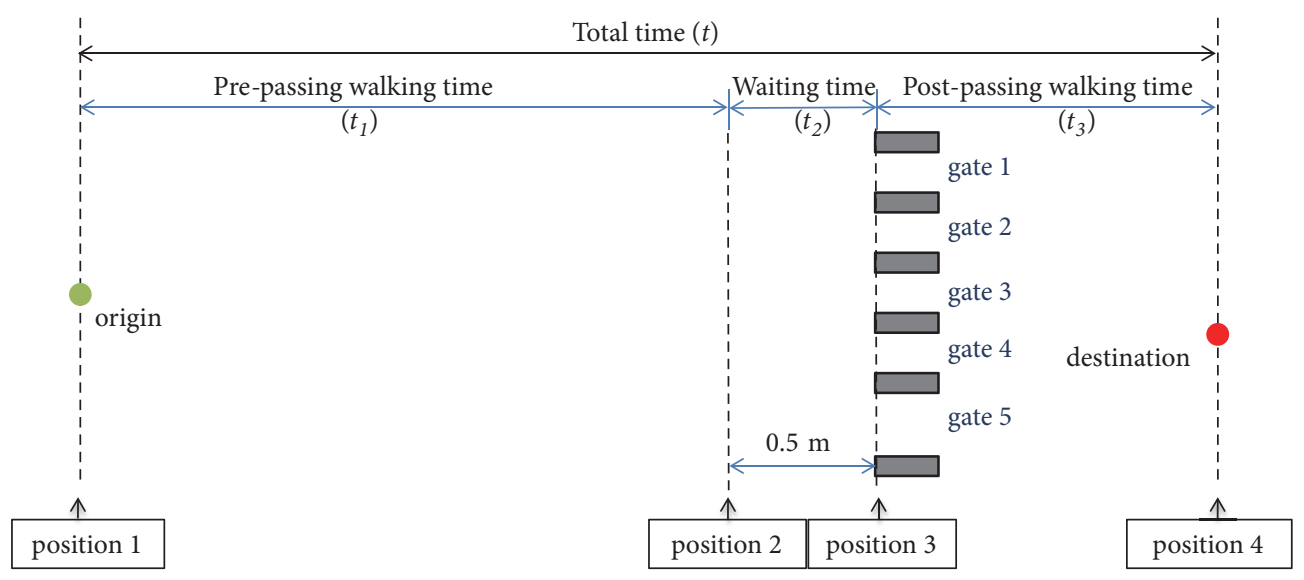

FIGURE 11: Measurement positions and travel time.

gates with the shortest distances (gate 4 and gate 5 ) have the largest passenger flows, accounting for $70 \%$. Due to the long distance, the number of passengers passed through gate 1 and gate 2 is small; especially gate 1 , only 4 persons passed. All passengers passed through the gates within $10 \mathrm{~s}$ to $343 \mathrm{~s}$.

As shown in Figure 10, in the asymmetric scenario, the passenger flow of each gate exhibits a more nonuniform distribution. To gain an insight into the distribution of passengers with different preference, the simulation results of the asymmetric scenario is a good case to illustrate this issue. As shown in Table 2, the distribution of the adventurous is obviously different from the others. For the conserved and the mild, more than 40 percentage of the pedestrian volume pass through the nearest gate 5 , and the total number of pedestrians passing through from the two nearest paths (gate 4 and 5) accounts for more than $70 \%$. In contrast, most of the adventurous pass through gate 3 and 4 , rather than the nearest gate 5 . This is thanks to the fact that the nearest gate 5 is always occupied by the conserved and the mild. As a result, in order to avoid congestion, the adventurous have to pass through the farther gates. In addition, there are a few of adventurous pedestrians pass through gate 1 , the farthest path. However, the conserved and the mild almost never choose gate 1 . Therefore, in the condition of a large number of passengers passing through ticket gates, the adventurous would make an important contribution to the balance of facilities utilization.

3.4. Passing Time. To clarify the effects of passenger preference on local route decision-making, we set four positions to record the travel time for each pedestrian during simulation. As shown in Figure 11, the first position is the origin point. The second is 0.5 -metre ahead to the gates. The third position is at the front edge of gates, where passengers are just walking into the gates. The last position is the destination of path. Then, four periods of time could be defined. Specifically, waiting time $\left(t_{2}\right)$ is the period for a passenger walking from position 2 to position 3 . It includes the time of walking, 
TABLE 2: Results of local route choice for different passengers.

\begin{tabular}{|c|c|c|c|c|c|c|}
\hline Temperament & gatel & gate 2 & gate 3 & gate 4 & gate 5 & Total \\
\hline adventurous & $4 \%$ & $12 \%$ & $28 \%$ & $31 \%$ & $24 \%$ & $100 \% / 67 p$ \\
\hline conserved & $1 \%$ & $9 \%$ & $17 \%$ & $31 \%$ & $41 \%$ & $100 \% / 70 p$ \\
\hline mild & $0 \%$ & $5 \%$ & $21 \%$ & $32 \%$ & $43 \%$ & $100 \% / 63 p$ \\
\hline Total & $2 \%$ & $9 \%$ & $22 \%$ & $32 \%$ & $36 \%$ & $100 \% / 200 \mathrm{p}$ \\
\hline
\end{tabular}
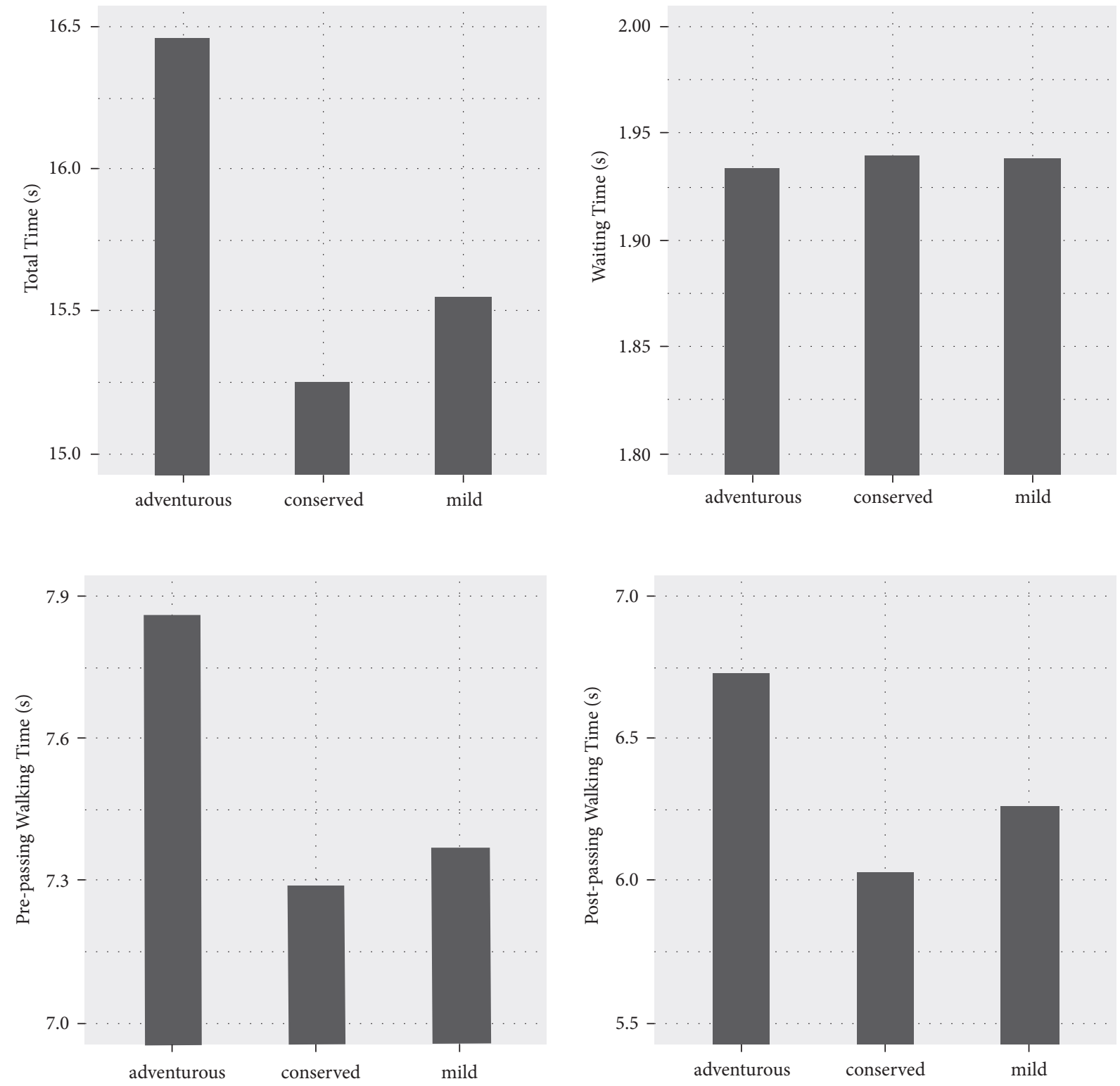

FIGURE 12: The passing times for different passengers.

scanning card, and queuing up. "Prepassing walking time" $\left(t_{1}\right)$ is the time taken to reach position 2 from the origin, and "postpassing walking time" $\left(t_{3}\right)$ is the time spent from position 3 to the destination. The total time spent in the whole journey from origin to destination is defined as $t$.

As the expected speed and scan-card time of different pedestrians follow the same distribution, the walking distances and queuing time of pedestrians can be compared using the four time periods. In the same way, take the results of the asymmetric scenario for example. As shown in Figure 12, the conserved pedestrians who prefer to wait at a congested gate spend more time on waiting and less time on walking. And for the pedestrians avoiding congestion, the results are completely the opposite. For the mild, the time they spend and the distance they traveled at each stage are always at the middle level. In particular, the adventurous 

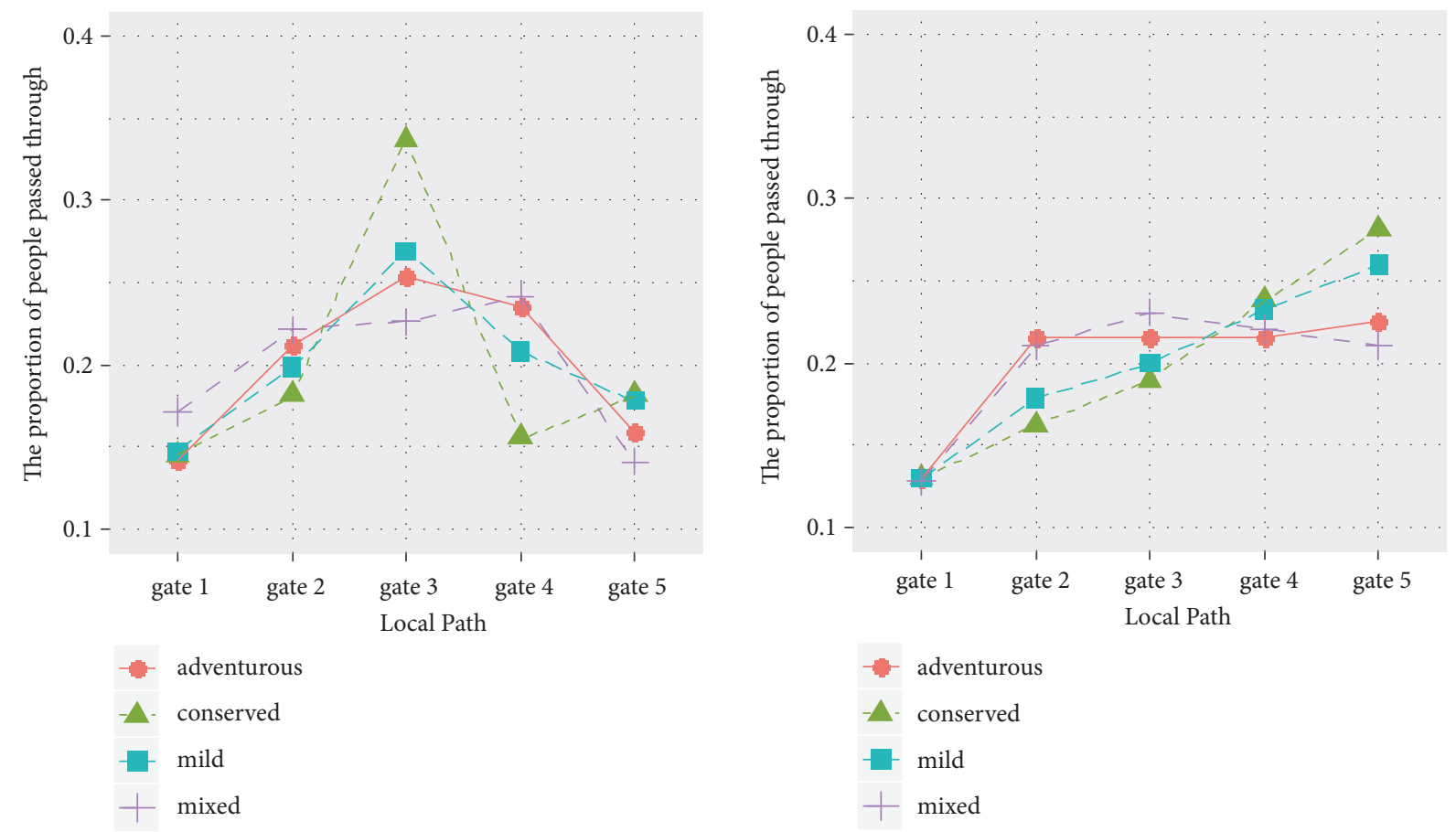

FIGURE 13: Four simulation cases with different choice preferences under symmetric layout (left) and asymmetric layout (right).

TABLE 3: The values of MD for different passengers.

\begin{tabular}{lcccc}
\hline MD & adventurous & conserved & mild & Total \\
\hline symmetric layout & 4.0 & 5.6 & 3.0 & 3.6 \\
asymmetric layout & 2.8 & 4.8 & 3.6 & 2.8 \\
\hline
\end{tabular}

passengers have the longest walking time (7.86 s before passing and $6.73 \mathrm{~s}$ after passing) and the shortest waiting time $(1.86 \mathrm{~s})$. This is in consistent with the hypothesis that they prefer to farther path to avoid queuing. On the contrary, the conserved passengers have the longest waiting time (1.94 s) but get to the destination faster by walking the shortest distance (total time is $15.26 \mathrm{~s}$ ). Although the adventurous wait $0.08 \mathrm{~s}$ less time than the conserved, they walk for $1.27 \mathrm{~s}$ more than the conserved. As a consequence, the adventurous spend $0.90 \mathrm{~s}$ on the whole journey more than the conserved on average. This is a kind of "fast is slow" phenomenon. With the given population size and passenger's composition, from an individual's perspective, it seems that waiting and passing through the near paths is the most time-saving strategy.

3.5. Individual Preference. The three kinds of personality in this paper actually map different choice preferences. In order to further investigate the differences among them, four simulation cases are established. In the first three cases, all pedestrians have the same character. In this way, the performance of a kind of passengers can be revealed solely without interference from other types of passengers. As a comparison, each pedestrian in Case 4 would have one of the three choice preferences randomly, while the passenger volume stays the same. To highlight the impact of preferences, the passenger load in the four cases is triple as much as the normal situation. The passenger flow passed through each gate is shown in Figure 13. In the four cases of the symmetric scenario, there are small passenger flow of the two marginal gates (gate 1 and 5). Meanwhile, the adventurous display a better balance at the three middle gates and the mild achieve a suboptimal balance, whereas the passenger size of the conserved pass through the middle gate 3 is significantly higher than other cases. In the asymmetric scenario, Case 2 (only the conserved) and Case 3 (only the mild) have similar results: the shorter distance a path is, the more passengers pass through. However, Case 1 (only the adventurous) has a different performance: the passenger population of each gate is much better balanced. Except for the farthest gate 1, the proportions of the adventurous pass through each gate are almost the same.

To evaluate the passenger distribution, the mean deviation (MD, Equation (14)) is introduced as a coefficient of uniformity. The evaluation results are shown in Table 3. A larger value of MD means a more uneven distribution of passengers at each gate and a poorer utilization of the facilities. For instance, in the symmetric scenario, there is a seriously nonuniform distribution of the conserved passengers with the biggest value of MD (5.6). In the asymmetric scenario, the MD value of the adventurous is the smallest and the facilities 

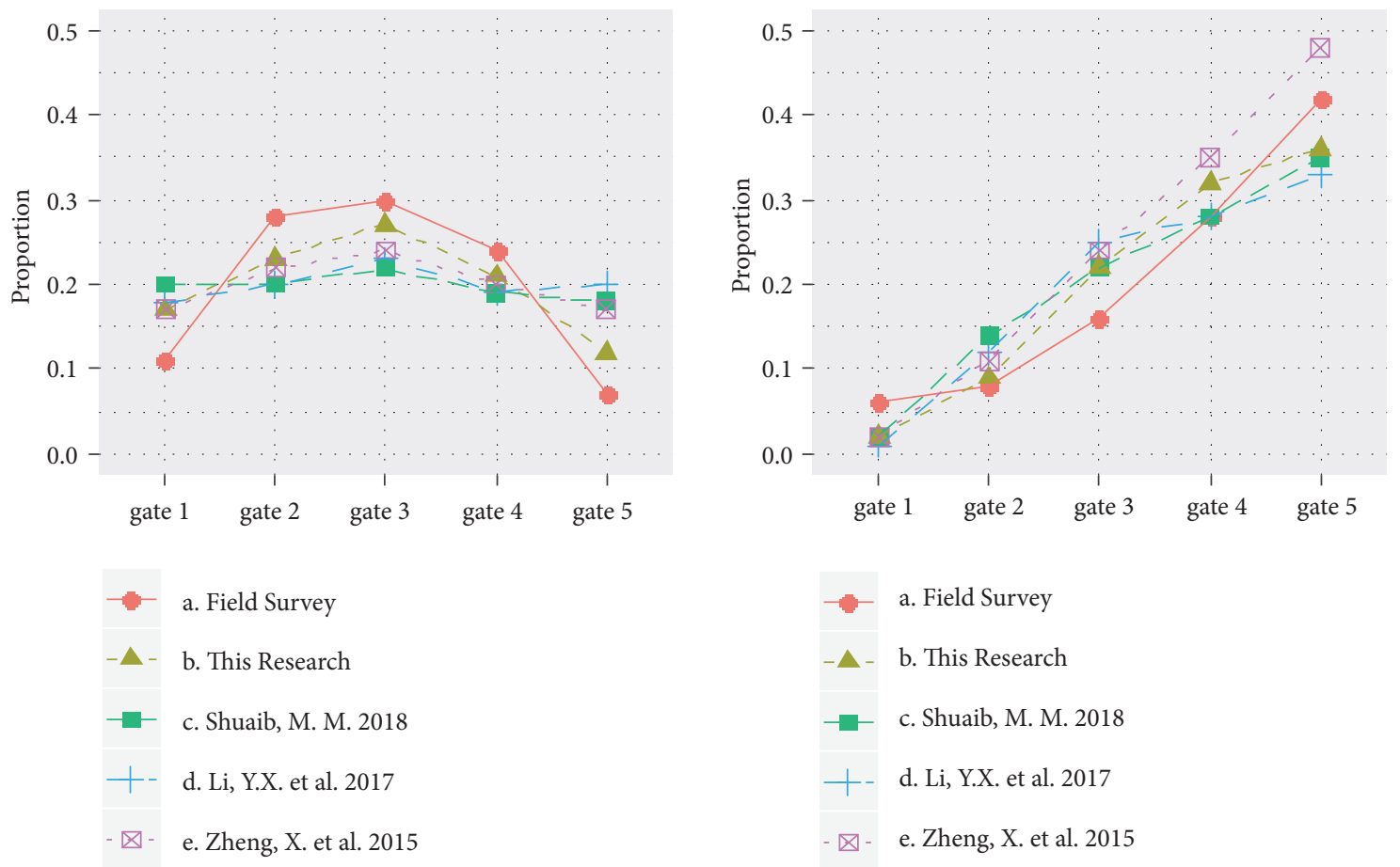

FIGURE 14: The survey data and simulation results under symmetric layout (left) and asymmetric layout (right).

are made best use in this case. Although the distributions of the mild and the conserved are similar, the mild present a better balance than the conserved. Actually, the MD values of the conserved are the biggest in the both scenarios and they are not conducive to make full utilization of facilities.

Interestingly, with mixed choice preferences, Case 4 is more in line with the practical condition. Both in the symmetric and asymmetric scenarios, Case 4 could achieve well balanced distributions. Furthermore, by a comparison with the simulation results in Section 3.3 (Figure 10), it can be found that, with the increased population size, the distribution of passenger flow of each gate is more balanced, and, as a result, the facility utilization is improved.

$$
\mathrm{MD}=\frac{1}{N} \sum_{j=1}^{M}\left|x_{j}-\bar{x}\right|
$$

where $\left(x_{1}, \ldots x_{j}, \ldots, x_{M}\right)$ is the proportion of passengers passing through each gate obtained in an experiment. $\bar{x}$ is the average value.

\section{Simulation Experiment}

In this section, some experiments are carried out to show the validity and stability of the model proposed in this paper. The techniques used to validate artificial pedestrian behaviours can be identified as three main streams [49]: the pedestrian dynamics descriptors, such as the fundamental diagram; the real data to validate the output of the models or simulation systems; and the visual realism and the way a human observer perceives in a simulation. Due to the fact that the main contributions of this paper focus on the mesolevel and macrolevel, we verified our model with the latter two techniques.

4.1. Simulation of LRP Model. As the core content of the layered navigation algorithm, the performance of the local route planning model determines the practical value of the navigation algorithm. As mentioned in the part of introduction, there are three kinds of alternative methods of node selection behaviour. They are based on utility, probability, and Logit model, respectively. Among each kind of method, a typical one with good performance is selected to compare with our LRP model and the field data. The compared methods were clearly stated in the literature and can be reproduced. Compared with the above models, the LRP model proposed in this paper makes further consideration of pedestrians' preferences, the process and motivation to make choice, and the uncertainties underlying in estimation and selection.

With the two typical layout scenarios of ticket gates, the simulation results are shown in Figure 14. In each experiment, the passenger size is the same as the survey. It can be seen that the results of the LRP model in this paper have better performance than the other methods, especially in the symmetric scenario. The simulation results can be evaluated by the mean relative error (MRE), expressed as Equation (15). The errors of different methods are indicated in Table 4. With the least values of MRE in both typical scenarios, the LRP model in this research is the best method to describe the node selection behaviour of ticket gates. By the LRP model 
TABLE 4: The values of MRE by different algorithms.

\begin{tabular}{lcccc}
\hline MRE & This Research & Shuaib, M. M. 2018 [7] & Li, Y. et al. 2017 [16] & Zheng, X. et al. 2015 [11] \\
\hline symmetric layout & 0.33 & 0.63 & 0.63 & 0.51 \\
asymmetric layout & 0.29 & 0.38 & 0.43 & 0.35 \\
\hline
\end{tabular}

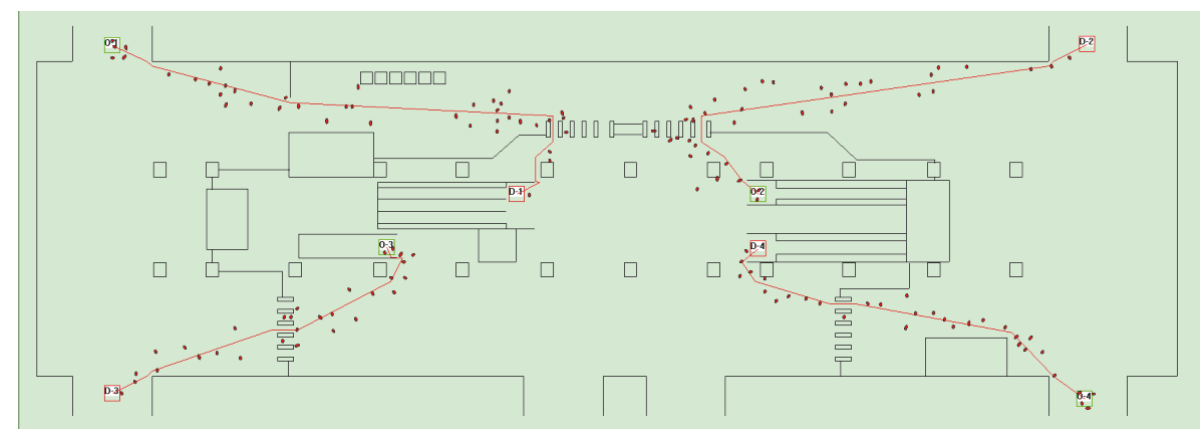

FIGURE 15: A screenshot of the simulation process.

we proposed, the difference between the simulation results and the field data is reduced.

$$
\operatorname{MRE}=\frac{1}{N} \sum_{j=1}^{N} \frac{\left|x_{j}-x_{j}^{0}\right|}{x_{j}^{0}}
$$

where $\left(x_{1}, \ldots x_{j}, \ldots, x_{N}\right)$ is the proportion of passengers passing through each gate obtained by simulation. $\left(x_{1}^{0}, \ldots, x_{j}^{0}\right.$, $\left.\ldots, x_{N}^{0}\right)$ is the proportion of the field data.

4.2. Simulation of Layered Navigation Model. In the layered navigation algorithm of this paper, the global path can be modified by the local route planning in real time according to the dynamic environment. It performs well in simulating the self-optimizing path choice behaviour of passengers in real world. Firstly, in order to illustrate the optimization function of the LRP model in the layered navigation algorithm, two experiments are carried out to simulate the movements of passengers at the hall in a subway station. In the first experiment, agents are only guided by the global route. While in the other experiment, the local route planning can be activated according to dynamic environment.

Take Qianmen Station on Line 8 of the Beijing Subway as a case. Four groups of passengers are loaded into each experiment. Passengers could enter the station from the upper-left and the lower-right entrances. Their arrival rate is assumed to follow a Poisson distribution with an average of 50 persons per minute. The passengers getting off a train will leave from the two exits located in the upper-right and the lower-left corner of the station. Alighting passengers show a pulse distribution as the train arrives. The trains would arrive at an interval of 2 minutes. There are sixty alighting passengers who would continue to reach the ticket gates in one minute.

The experiments are carried out by the simulation tool we developed. According to the framework shown in Figure 3, the static global routes for each passenger group are firstly generated by an "initialization route" module. They are shown as the red polylines in Figure 15. Due to the similar activity planning of pedestrians in a subway station, it is reasonable to make the same global route for the pedestrians who have the same OD pair. In particular, the local route areas shown in Figure 5 should be set for the places needing local route choice. For example, when a pedestrian gets to the ticket gate, the local route plan will be activated. Figure 15 shows a screenshot after the simulation running for 54 seconds. The red ellipses represent agents.

The level of service (LOS) is an important criterion to evaluate a metro station. LOS is introduced by Fruin [50] to assess pedestrian facilities for the first time. Based on that, Transit Capacity and Quality of Service Manual takes the comfort and convenience of pedestrian into account and put forward a method to assessment the LOS, as shown in Table 5. By the "cluster display mode" of our simulation tool, the LOS of local area can be marked in the map during simulation. Figure 16 displays the screenshots of simulation. In Figure 16(a), passengers just walk along the global route and do not consider local congestion. This caused the crowdedness at the entrance of the ticket gates and escalators. At these places, the LOS is always at level E. In contrast, when the agents can respond to dynamic surroundings by the local route algorithm, the congestions at bottleneck locations are relieved effectively and the LOS is decreased in all local areas. In most cases, the LOS is not above level C. Level E only appears occasionally for a short time and could be adjusted by the self-organization of pedestrians quickly. In conclusion, the model just based on the global route generates unrealistic behaviour in the case of congestion. However, the model including the LRP allows describing the redistribution of the pedestrians over the gates.

In order to further demonstrate the effect of the hybrid navigation algorithm proposed in this paper, we also simulate the passenger movements in the above subway station by commercial software VISSIM. It is widely used in the research of pedestrian simulation. The settings of building 
TABle 5: Service level.

\begin{tabular}{lcccccc}
\hline Service level & A & B & C & D & E & F \\
\hline Personal space $\left(\mathrm{m}^{2} / \mathrm{p}\right)$ & $\geq 3.3$ & $2.3-3.3$ & $1.4-2.3$ & $0.9-1.4$ & $0.5-0.9$ & $<0.5$ \\
Density $\left(\mathrm{p} / \mathrm{m}^{2}\right)$ & $\leq 0.3$ & $0.3-0.4$ & $0.4-0.7$ & $0.7-1.1$ & $1.1-2.0$ & $>2.0$ \\
\hline
\end{tabular}

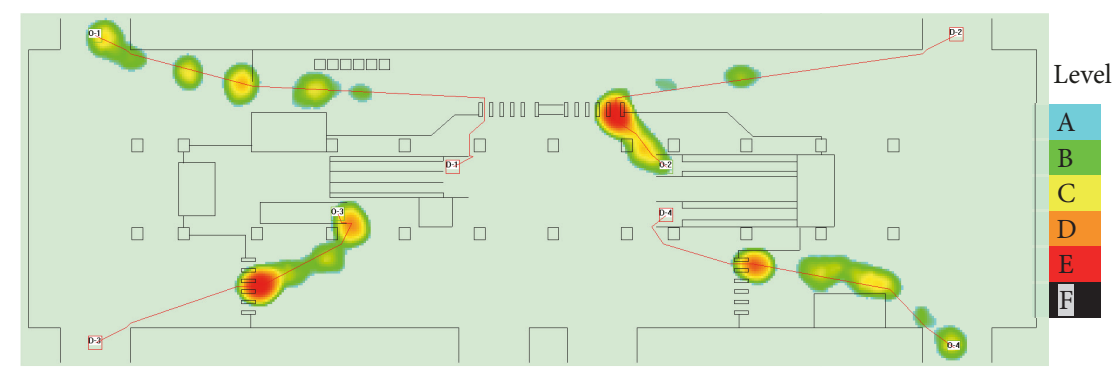

(a)

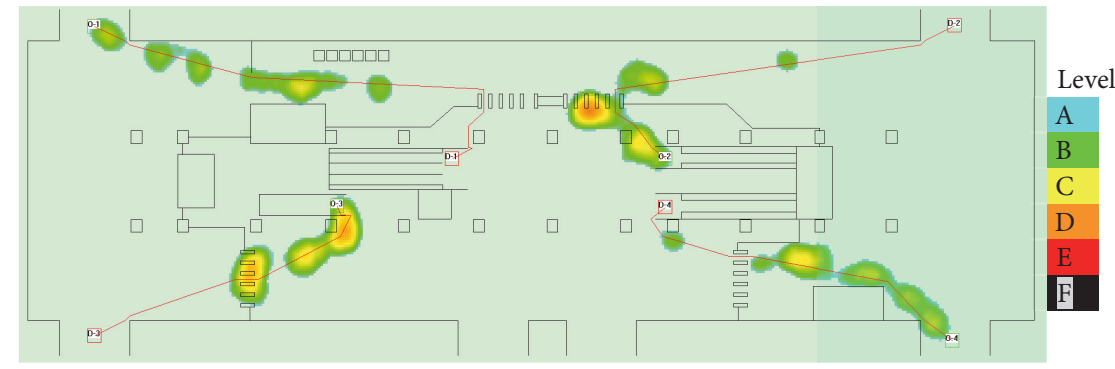

(b)

FIgURe 16: Mapped LOS in a station (T=26s), (a) only global route planning and (b) two-level route planning.

TABLE 6: Comparison of local route simulation results.

\begin{tabular}{lccc}
\hline Average value & Distance $(\mathrm{m})$ & Travel time $(\mathrm{s})$ & Speed $(\mathrm{m} / \mathrm{s})$ \\
\hline VISSIM & 19.171 & 53.449 & 0.359 \\
the developed tool & 27.891 & 30.275 & 0.922 \\
\hline
\end{tabular}

environment and population size are the same as Figure 15. The experimental results of our tool and that of VISSIM are shown in Table 6 . In our model, the local route planning based on the estimated time may increase the walking distance, but produce faster speed and less total travel time. In this respect, the model presented in this paper has better performances.

At the tactical level, our tool realizes a dynamic feedback and real-time planning, which could improve the utilization of facilities and reduce travel time. Passengers could choose the ticket gate rationally without crowding, as shown in Figure 17(a). However, although some commercial software has introduced local route planning into simulation algorithm, the effect on uniform use of facilities is not very ideal. Its route planning tends to be very mechanical and leads to distorted behaviour (Figure 17(b)).

\section{Conclusion}

We presented a novel approach to deal with the problem of route planning in dynamic environments. The method is based on a seamlessly transition between global planning and local planning. In order to generate a no-collision global route, we made a correction to the visibility graph method by expanding the obstacles to avoid the overlap of pedestrian's body with obstacles. We have further introduced a new and effective local route planning model based on estimated passing time. This approach results in smoother crowd movement and exhibits an agent's tendency to avoid congestion that is often observed in real crowds. The uncertainties existing in the process of node selection, such as the estimation of factors, the randomness of decision-making, and the subjective preferences of different passengers are fully integrated into the model. In its application to the selection of ticket gates in subway stations, the activity of card feeding is embedded. The whole process of selection and reselection is simplified into three stages and the differences of passengers' focuses during them are captured. Compared to previous navigation algorithms, it is demonstrated that our approach has better performances in multipath choices during trip. The agents in our simulation could produce faster speed and less travel time. Furthermore, our model is founded on cognitive science work on human locomotion, which can be combined with various classical methods for navigation in dynamic environments and open interesting perspectives for realistic simulation purposes.

In this paper, the route planning model is proposed based on the ticket gates in subway station. It can be extended 


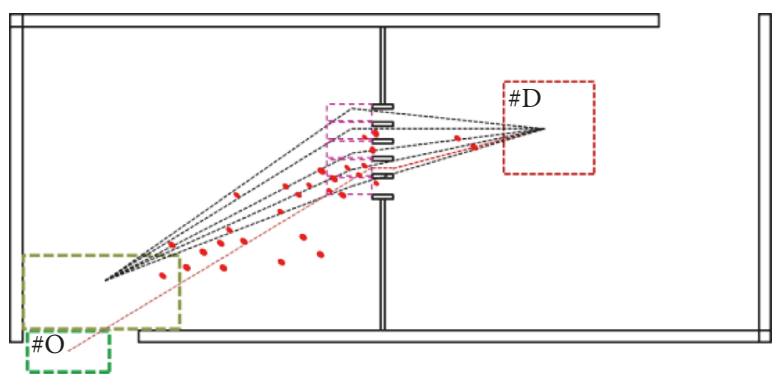

(a)

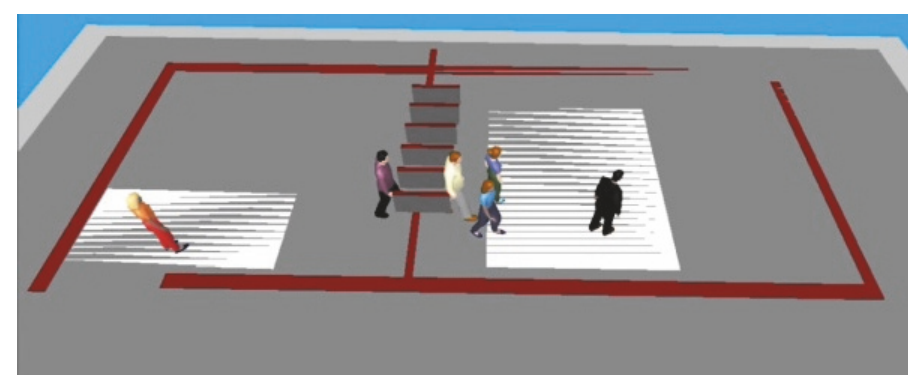

(b)

FIGURE 17: A simulation snapshot by (a) our tool and (b) VISSIM.

to other similar scenarios of exit selection and multipath planning. In the future, the route planning model can be integrated with other strategic and operational methods to simulate the movement of pedestrians. Moreover, the route planning model will have potential to be applied to many other simulation cases, such as emergency evacuation and disaster response systems.

\section{Data Availability}

The empirical data used to support the findings of this study can be found in the section of "Model Application and Discussion". The "simulation results" data used to support the findings of this study are included within the article.

\section{Conflicts of Interest}

The authors declare that there are no conflicts of interest regarding the publication of this paper.

\section{Acknowledgments}

This work is supported by the National Natural Science Foundation of China (No. 71871027 and No. 51578054).

\section{References}

[1] S. P. Hoogendoorn, P. Bovy, and W. Daamen, "Microscopic pedestrian wayfinding and dynamics modelling," in Proceedings of the International Conference on Pedestrian and Evacuation Dynamics, pp. 123-155, 2002.

[2] H. Kim and S. Han, "Crowd evacuation simulation using active route choice model based on human characteristics," Simulation Modelling Practice and Theory, vol. 87, pp. 369-378, 2018.

[3] A. Cuesta, O. Abreu, A. Balboa, and D. Alvear, "Real-time evacuation route selection methodology for complex buildings," Fire Safety Journal, vol. 91, pp. 947-954, 2017.

[4] P. M. Kielar, D. H. Biedermann, A. Kneidl, and A. Borrmann, "A unified pedestrian routing model for graph-based wayfinding built on cognitive principles," Transportmetrica A: Transport Science, vol. 14, no. 5, pp. 406-432, 2018.

[5] F. Farina, D. Fontanelli, A. Garulli, A. Giannitrapani, and D. Prattichizzo, "Walking ahead: The headed social force model," PLoS ONE, vol. 12, no. 1, Article ID e0169734, 2017.
[6] Y. Hong, D. Li, Q. Wu, and $\mathrm{H}$. Xu, "Dynamic route network planning problem for emergency evacuation in restrictedspace scenarios," Journal of Advanced Transportation, vol. 2018, Article ID 4295419, 13 pages, 2018.

[7] M. M. Shuaib, "Incorporating intelligence for typical evacuation under the threat of fire spreading," Safety Science, vol. 106, pp. $1-9,2018$.

[8] M. Shuaib and Z. Zainuddin, "Incorporating intelligence into exit choice model for typical evacuation," Sains Malaysiana, vol. 46, no. 10, pp. 1997-2005, 2017.

[9] A. U. Kemloh Wagoum, A. Tordeux, and W. Liao, "Understanding human queuing behaviour at exits: an empirical study," Royal Society Open Science, vol. 4, no. 1, Article ID 160896, 2017.

[10] Z. Li, "An emergency exits choice preference model based on characteristics of individual diversity," Advances in Mechanical Engineering, vol. 9, no. 4, p. 168781401769354, 2017.

[11] X. Zheng, H.-Y. Li, L.-Y. Meng, X.-Y. Xu, and X. Chen, "Improved social force model based on exit selection for microscopic pedestrian simulation in subway station," Journal of Central South University, vol. 22, no. 11, pp. 4490-4497, 2015.

[12] M. Tanaka, D. Yanagisawa, and K. Nishinari, "Exclusive queueing model including the choice of service windows," Physica A: Statistical Mechanics and its Applications, vol. 490, pp. 14811492, 2018.

[13] R. Lovreglio, A. Fonzone, L. dell'Olio, and D. Borri, "A study of herding behaviour in exit choice during emergencies based on random utility theory," Safety Science, vol. 82, pp. 421-431, 2016.

[14] R.-Y. Guo and H.-J. Huang, "Logit-based exit choice model of evacuation in rooms with internal obstacles and multiple exits," Chinese Physics B, vol. 19, no. 3, Article ID 030501, 2010.

[15] D. Duives and H. Mahmassani, "Exit choice decisions during pedestrian evacuations of buildings," Transportation Research Record, no. 2316, pp. 84-94, 2012.

[16] Y. Li, H. Jia, J. Li, J. Gong, and K. Sun, "Pedestrian evacuation behavior analysis and simulation in multi-exits case," International Journal of Modern Physics C, vol. 28, no. 10, Article ID 1750128, 15 pages, 2017.

[17] B. Liu, H. Liu, H. Zhang, and X. Qin, "A social force evacuation model driven by video data," Simulation Modelling Practice and Theory, vol. 84, pp. 190-203, 2018.

[18] K. Rendón Rozo, J. Arellana, A. Santander-Mercado, and M. Jubiz-Diaz, "Modelling building emergency evacuation plans considering the dynamic behaviour of pedestrians using agentbased simulation," Safety Science, vol. 113, pp. 276-284, 2019.

[19] X. Chen, H. Li, J. Miao, S. Jiang, and X. Jiang, "A multiagentbased model for pedestrian simulation in subway stations," 
Simulation Modelling Practice and Theory, vol. 71, pp. 134-148, 2017.

[20] M. Haghani and M. Sarvi, "Social dynamics in emergency evacuations: disentangling crowd's attraction and repulsion effects," Physica A: Statistical Mechanics and its Applications, vol. 475, pp. 24-34, 2017.

[21] M. Haghani and M. Sarvi, "Stated and revealed exit choices of pedestrian crowd evacuees," Transportation Research Part B: Methodological, vol. 95, pp. 238-259, 2017.

[22] M. Kinateder, B. Comunale, and W. H. Warren, "Exit choice in an emergency evacuation scenario is influenced by exit familiarity and neighbor behavior," Safety Science, vol. 106, pp. 170-175, 2018.

[23] M. Haghani and M. Sarvi, "Human exit choice in crowded built environments: Investigating underlying behavioural differences between normal egress and emergency evacuations," Fire Safety Journal, vol. 85, pp. 1-9, 2016.

[24] R. Lovreglio, A. Fonzone, and L. dell'Olio, "A mixed logit model for predicting exit choice during building evacuations," Transportation Research Part A: Policy and Practice, vol. 92, pp. 59-75, 2016.

[25] X. Song, J. Sun, H. Xie, Q. Li, Z. Wang, and D. Han, “Characteristic time based social force model improvement and exit assignment strategy for pedestrian evacuation," Physica A: Statistical Mechanics and its Applications, vol. 505, pp. 530-548, 2018.

[26] H. Liu, B. Xu, D. Lu, and G. Zhang, "A path planning approach for crowd evacuation in buildings based on improved artificial bee colony algorithm," Applied Soft Computing, vol. 68, pp. 360376, 2018.

[27] R.-Y. Guo, "Potential-based dynamic pedestrian flow assignment," Transportation Research Part C: Emerging Technologies, vol. 91, pp. 263-275, 2018.

[28] M. Stubenschrott, T. Matyus, H. Schrom-Feiertag, C. Kogler, and S. Seer, "Route-choice modeling for pedestrian evacuation based on infrastructure knowledge and personal preferences," Transportation Research Record, vol. 2623, no. 1, pp. 82-89, 2017.

[29] T. Q. Tang, Y. X. Shao, L. Chen, and H. Y. Shang, "Modeling passengers' boarding behavior at the platform of high speed railway station," Journal of Advanced Transportation, vol. 2017, Article ID 4073583, 11 pages, 2017.

[30] T.-Q. Tang, Y.-X. Shao, and L. Chen, "Modeling pedestrian movement at the hall of high-speed railway station during the check-in process," Physica A: Statistical Mechanics and its Applications, vol. 467, pp. 157-166, 2017.

[31] Y. Cheng and X. Zheng, "Effect of uncertainty on cooperative behaviors during an emergency evacuation," Communications in Nonlinear Science and Numerical Simulation, vol. 66, pp. 216225, 2019.

[32] D. R. Bish and H. D. Sherali, "Aggregate-level demand management in evacuation planning," European Journal of Operational Research, vol. 224, no. 1, pp. 79-92, 2013.

[33] Y. Lv, X. D. Yan, W. Sun, and Z. Y. Gao, "A risk-based method for planning of bus-subway corridor evacuation under hybrid uncertainties," Reliability Engineering \& System Safety, vol. 139, pp. 188-199, 2015.

[34] S. P. Hoogendoorn and P. H. L. Bovy, "Pedestrian route-choice and activity scheduling theory and models," Transportation Research Part B: Methodological, vol. 38, no. 2, pp. 169-190, 2004.
[35] H.-H. Tian, Y.-F. Wei, L.-Y. Dong, Y. Xue, and R.-S. Zheng, "Resolution of conflicts in cellular automaton evacuation model with the game-theory," Physica A: Statistical Mechanics and its Applications, vol. 503, pp. 991-1006, 2018.

[36] S. Cao, L. Fu, and W. Song, "Exit selection and pedestrian movement in a room with two exits under fire emergency," Applied Mathematics and Computation, vol. 332, pp. 136-147, 2018.

[37] R. E. Tarjan, “A unified approach to path problems," Journal of the ACM, vol. 28, no. 3, pp. 577-593, 1981.

[38] N. WEI, Research on globalroute planning for virtual human [Master thesis], JiangSu University, China, 2007.

[39] E. Palma-Villalon and P. Dauchez, "World representation and path planning for a mobile robot," Robotica, vol. 6, no. 1, pp. 3540, 1988.

[40] L. G. Sun and Q. X. Shi, "Algorithm of pedestrians' route planning based on discrete potential field," Transport Standardization, vol. 23, pp. 25-27, 2009.

[41] A. Abdelghany, K. Abdelghany, H. Mahmassani, H. Al-Ahmadi, and W. Alhalabi, "Modeling the evacuation of large-scale crowded pedestrian facilities," Transportation Research Record, no. 2198, pp. 152-160, 2010.

[42] R. Lovreglio, D. Borri, L. Dell'Olio, and A. Ibeas, "A discrete choice model based on random utilities for exit choice in emergency evacuations," Safety Science, vol. 62, pp. 418-426, 2014.

[43] L. Zhang, M. Liu, X. Wu, and S. M. AbouRizk, "Simulationbased route planning for pedestrian evacuation in metro stations: a case study," Automation in Construction, vol. 71, no. 2, pp. 430-442, 2016.

[44] M. Nasir, C. P. Lim, S. Nahavandi, and D. Creighton, "Prediction of pedestrians routes within a built environment in normal conditions," Expert Systems with Applications, vol. 41, no. 10, pp. 4975-4988, 2014.

[45] W. Liao, A. U. K. Wagoum, and N. W. F. Bode, "Route choice in pedestrians: determinants for initial choices and revising decisions," Journal of the Royal Society Interface, vol. 14, no. 127, 2017.

[46] M. Haghani and M. Sarvi, "Pedestrian crowd tactical-level decision making during emergency evacuations," Journal of Advanced Transportation, vol. 50, no. 8, pp. 1870-1895, 2016.

[47] Y. Han, H. Liu, and P. Moore, "Extended route choice model based on available evacuation route set and its application in crowd evacuation simulation," Simulation Modelling Practice and Theory, vol. 75, pp. 1-16, 2017.

[48] J.-J. Hsu and J. C. Chu, "Long-term congestion anticipation and aversion in pedestrian simulation using floor field cellular automata," Transportation Research Part C: Emerging Technologies, vol. 48, pp. 195-211, 2014.

[49] F. Martinez-Gil, M. Lozano, I. García-Fernández, and F. Fernández, "Modeling, evaluation, and scale on artificial pedestrians," ACM Computing Surveys, vol. 50, no. 5, p. 72, 2017.

[50] J. J. Fruin, PedestrIan Planning and Design, Metropolitan Association of Urban Designers \& Environmental Planners, 1971. 


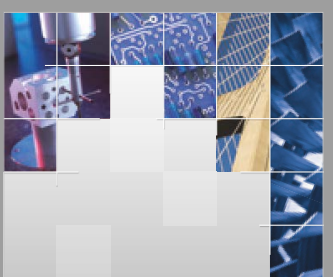

\section{Enfincering}
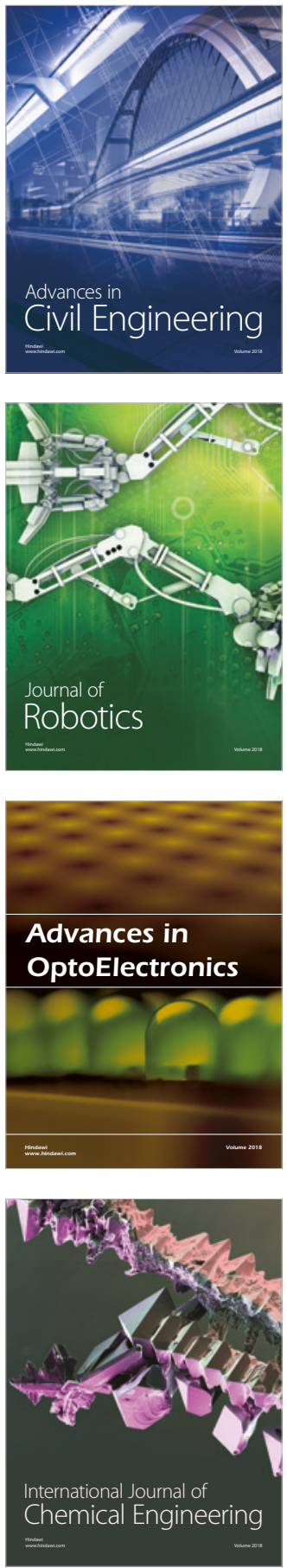

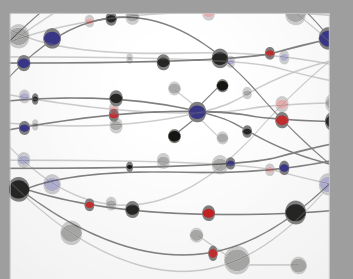

\section{Rotating \\ Machinery}

The Scientific World Journal

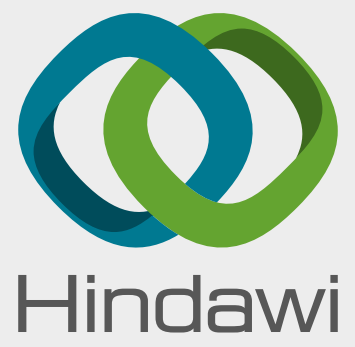

Submit your manuscripts at

www.hindawi.com
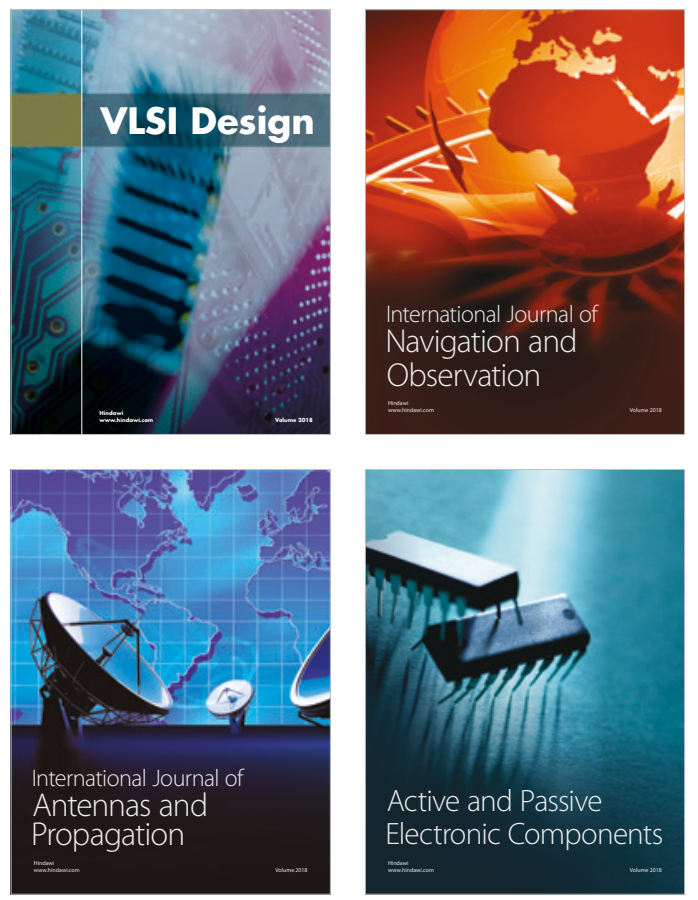
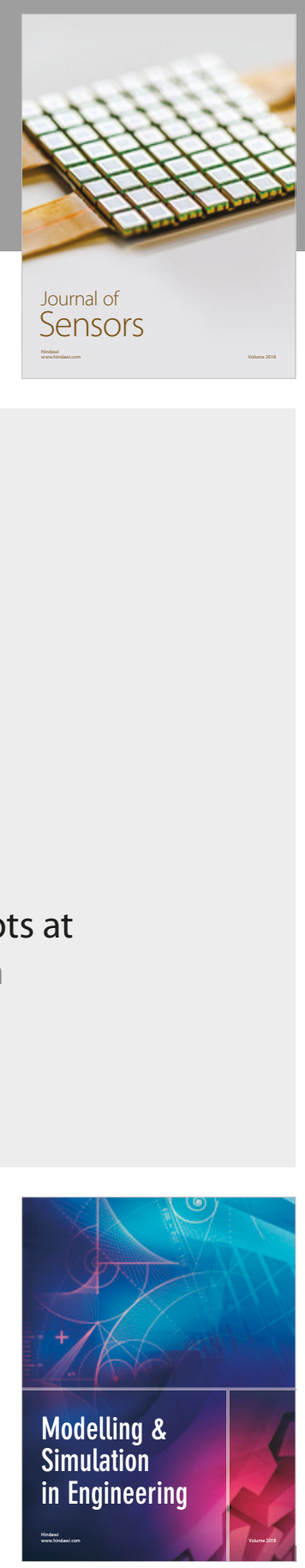

\section{Advances \\ Multimedia}
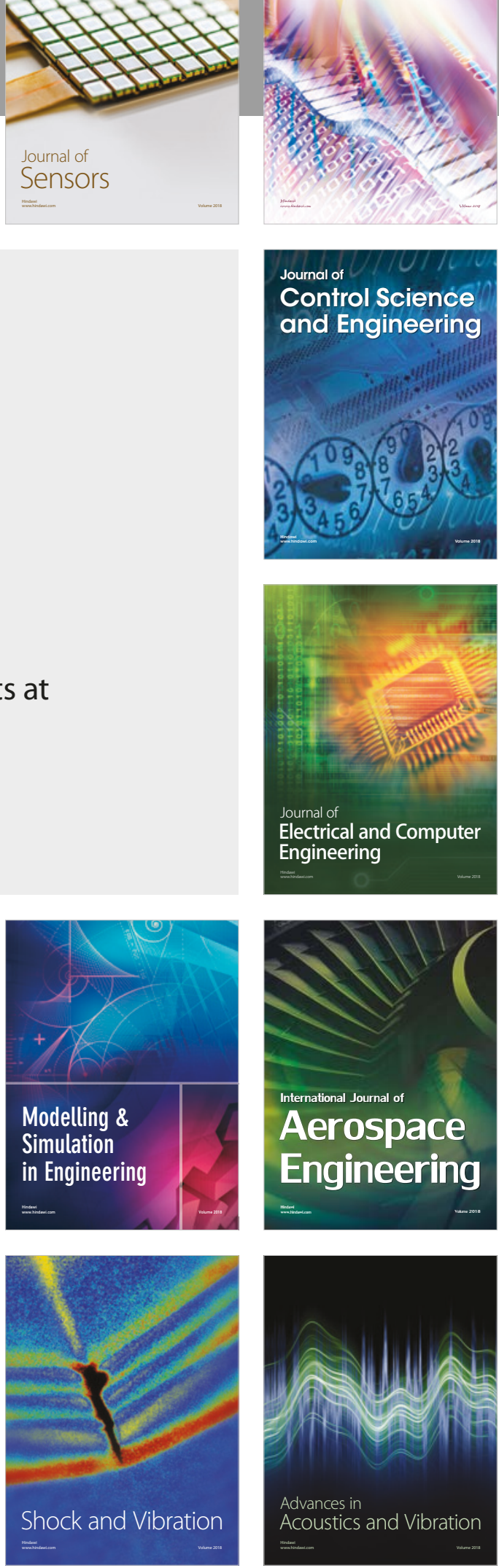